\title{
A family of pseudo-Anosov braids with small dilatation
}

\author{
ERIKO HIRONAKA \\ EIKO KIN
}

\begin{abstract}
This paper describes a family of pseudo-Anosov braids with small dilatation. The smallest dilatations occurring for braids with 3,4 and 5 strands appear in this family. A pseudo-Anosov braid with $2 g+1$ strands determines a hyperelliptic mapping class with the same dilatation on a genus- $g$ surface. Penner showed that logarithms of least dilatations of pseudo-Anosov maps on a genus- $g$ surface grow asymptotically with the genus like $1 / g$, and gave explicit examples of mapping classes with dilatations bounded above by $\log 11 / \mathrm{g}$. Bauer later improved this bound to $\log 6 / \mathrm{g}$. The braids in this paper give rise to mapping classes with dilatations bounded above by $\log (2+\sqrt{3}) / g$. They show that least dilatations for hyperelliptic mapping classes have the same asymptotic behavior as for general mapping classes on genus- $g$ surfaces.
\end{abstract}

37E30, 57M50

\section{Introduction}

In this paper, we study a family of generalizations of these examples to arbitrary numbers of strands. Let $\mathcal{B}(D, s)$ denote the braid group on $D$ with $s$ strands, where $D$ denotes a 2-dimensional closed disk. First consider the braids $\beta_{m, n}$ in $\mathcal{B}(D, m+n+1)$ given by

$$
\beta_{m, n}=\sigma_{1} \ldots \sigma_{m} \sigma_{m+1}^{-1} \ldots \sigma_{m+n}^{-1} .
$$

Matsuoka's example [22] appears as $\beta_{1,1}$, and Ko, Los and Song's example [18] as $\beta_{2,1}$. For any $m, n \geq 1, \beta_{m, n}$ is pseudo-Anosov (Theorem 3.9). The dilatations of $\beta_{m, m}$ coincide with those found by Brinkmann [7] (see also Section 4.2), who also shows that the dilatations arising in this family can be made arbitrarily close to 1 .

It turns out that one may find smaller dilatations by passing a strand of $\beta_{m, n}$ once around the remaining strands. As a particular example, we consider the braids $\sigma_{m, n}$ defined by taking the rightmost-strand of $\beta_{m, n}$ and passing it counter-clockwise once around the remaining strands. Figure 1 gives an illustration of $\beta_{m, n}$ and $\sigma_{m, n}$. The braid $\sigma_{1,3}$ is conjugate to Ham and Song's braid $\sigma_{1} \sigma_{2} \sigma_{3} \sigma_{4} \sigma_{1} \sigma_{2}$. For $|m-n| \leq 1$, we show that $\sigma_{m, n}$ is periodic or reducible. Otherwise $\sigma_{m, n}$ is pseudo-Anosov with 


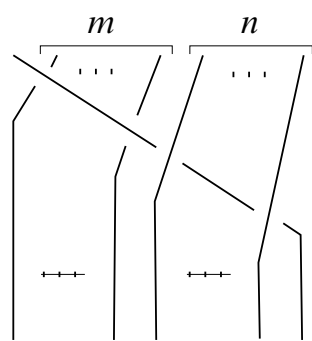

(a)

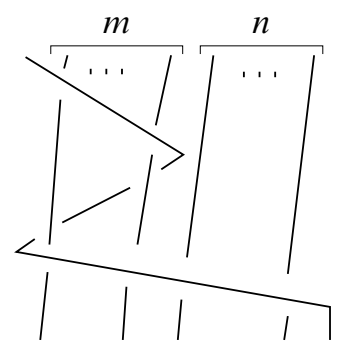

(b)

Figure 1: Braids (a) $\beta_{m, n}$ and (b) $\sigma_{m, n}$

dilatation strictly less than the dilatation of $\beta_{m, n}$ (Theorem 3.11, Corollary 3.32). The dilatations of $\sigma_{g-1, g+1}(g \geq 2)$ satisfy the inequality

$$
\lambda\left(\sigma_{g-1, g+1}\right)^{g}<2+\sqrt{3}
$$

(Proposition 3.36).

Let $\mathcal{M}_{g}^{s}$ denote the set of mapping classes (or isotopy classes) of homeomorphisms on the closed orientable genus- $g$ surface $F_{g}$ set-wise preserving $s$ points. We denote $\mathcal{M}_{g}^{0}$ by $\mathcal{M}_{g}$. For any subset $\Gamma \subset \mathcal{M}_{g}^{s}$, define $\lambda(\Gamma)$ to be the least dilatation among pseudo-Anosov elements of $\Gamma$, and let $\delta(\Gamma)$ be the logarithm of $\lambda(\Gamma)$. For the braid group $\mathcal{B}(D, s)$, and any subset $\Gamma \subset \mathcal{B}(D, s)$, define $\lambda(\Gamma)$ and $\delta(\Gamma)$ in a similar way. By a result of Penner [25] (see also McMullen [23]), $\delta\left(\mathcal{M}_{g}\right) \asymp \frac{1}{g}$.

An element of $\mathcal{M}_{g}$ is called hyperelliptic if it commutes with an involution $\iota$ on $F_{g}$ such that the quotient of $F_{g}$ by $\iota$ is $S^{2}$. Let $\mathcal{M}_{g \text {,hyp }} \subset \mathcal{M}_{g}$ denote the subset of hyperelliptic elements of $\mathcal{M}_{g}$. Any pseudo-Anosov braid on $2 g+1$ strands determines a hyperelliptic element of $\mathcal{M}_{g}$ with the same dilatation (Proposition 2.10). Thus, (1) implies:

Theorem 1.1 For $g \geq 2$,

$$
\delta\left(\mathcal{M}_{g}\right) \leq \delta\left(\mathcal{M}_{g, \text { hyp }}\right) \leq \delta(\mathcal{B}(D, 2 g+1))<\frac{\log (2+\sqrt{3})}{g} .
$$

This improves the upper bounds on $\delta\left(\mathcal{M}_{g}\right)$ found by Penner $\left(\frac{\log 11}{g}\right)$ [25] and Bauer $\left(\frac{\log 6}{g}\right)$ [1]. Theorem 1.1 shows the following.

Theorem 1.2 For $g \geq 2$,

$$
\delta(\mathcal{B}(D, 2 g+1)) \asymp \frac{1}{g} \quad \text { and } \quad \delta\left(\mathcal{M}_{g, \text { hyp }}\right) \asymp \frac{1}{g} .
$$


This paper is organized as follows. Section 2 reviews basic terminology and results on mapping class groups. In Section 3, we determine the Thurston-Nielsen types of $\beta_{m, n}$ and $\sigma_{m, n}$ by finding efficient graph maps for their monodromy actions following Bestvina and Handel [2]. We observe that the associated train tracks have "star-like" components, and their essential forms don't depend on $m$ and $n$ (Figures 20 and 21). To find bounds and inequalities among the dilatations, we apply the notion of Salem-Boyd sequences $[4 ; 28]$, and relate the similar forms of the efficient graph maps for $\beta_{m, n}$ and $\sigma_{m, n}$ to similar forms for characteristic polynomials of the dilatations. In particular, we show that the least dilatation that occurs among $\beta_{m, n}$ and $\sigma_{m, n}$ for $m+n=2 g(g \geq 2)$ is realized by $\sigma_{g-1, g+1}$, and find bounds for $\lambda\left(\sigma_{g-1, g+1}\right)$ yielding the inequality (1). Section 4 discusses the problem of determining the least dilatations of special subclasses of pseudo-Anosov maps. In Section 4.1, we briefly describe the relation between the forcing relation on braid types and dilatations, and show how $\sigma_{m, n}$ arise as the braid types of periodic orbits of the Smale-horseshoe map. In Section 4.2, we consider pseudo-Anosov maps arising as the monodromy of fibered links, and relate our examples to those of Brinkmann.

\section{Acknowledgements}

The authors thank Hiroyuki Minakawa for valuable discussions, and an algebraic trick that improved our original upper bound for $\lambda\left(\sigma_{g-1, g+1}\right)$. The first author thanks the J S P S, Osaka University and host Makoto Sakuma for their hospitality and support during the writing of this paper. The second author is grateful for the financial support provided by the research fellowship of the 21st century COE program in Kyoto University.

\section{Preliminaries}

In this section, we review basic definitions and properties of braids (Section 2.1), mapping class groups (Section 2.2), spectra (Section 2.3), and a criterion of the pseudoAnosov property (Section 2.4). Some results are well-known, and more complete expositions can be found in the articles by Bestvina-Handel [2] and Fathi-LaudenbachPoenaru [10], and the books by Birman [3], and Casson-Bleiler [8]. We include them here for the convenience of the reader.

\subsection{Braids}

Let $F$ be a compact orientable surface with $s$ marked points $\mathcal{S}=\left\{p_{1}, \ldots, p_{s}\right\} \subset \operatorname{int}(F)$, the interior of $F$. A braid representative $\beta$ on $F$ is the images of continuous maps

$$
f_{p_{1}}, \ldots, f_{p_{s}}: I=[0,1] \rightarrow F \times I,
$$

Algebraic $8 \mathcal{G}$ Geometric Topology, Volume 6 (2006) 
satisfying for $i=1, \ldots, s$,

(B1) $f_{p_{i}}(0)=p_{i} \times 0$,

(B2) $f_{p_{i}}(1) \in \mathcal{S} \times 1$,

(B3) $f_{p_{i}}(t) \in F \times t$ for $t \in I$, and

(B4) $f_{p_{i}}(t) \neq f_{p_{j}}(t)$ for $t$ and $i \neq j$.

Define the product of two braid representatives to be their concatenation. Let $\mathcal{B}(F ; \mathcal{S})$ be the set of braid representatives up to ambient isotopy fixing the boundary of $F$ point-wise. The above definition of product determines a well-defined group structure on $\mathcal{B}(F ; \mathcal{S})$, and the group is called the braid group on $F$.

For any partition $\mathcal{S}=\mathcal{S}_{1} \cup \cdots \cup \mathcal{S}_{r}$, let $\mathcal{B}\left(F ; \mathcal{S}_{1}, \ldots, \mathcal{S}_{r}\right)$ be the subgroup of $\mathcal{B}(F ; \mathcal{S})$ consisting of braids $\left(f_{p_{1}}, \ldots, f_{p_{s}}\right)$ satisfying for all $p \in \mathcal{S}_{j}(j \in\{1, \ldots, r\}), f_{p}(1) \in$ $\mathcal{S}_{j}$.

In the rest of this section, we assume that $F$ is either a disk $D$ or a sphere $S^{2}$. Then the braid group $\mathcal{B}(F ; \mathcal{S})$ has generators $\sigma_{1}, \ldots, \sigma_{s-1}$, where $\sigma_{i}$ is the braid shown in Figure 2. When $F=D, \mathcal{B}(D ; \mathcal{S})$ is called the Artin braid group and has finite presentation

$$
\left\langle\sigma_{1}, \ldots, \sigma_{s-1}: \sigma_{i} \sigma_{i+1} \sigma_{i}=\sigma_{i+1} \sigma_{i} \sigma_{i+1}, \sigma_{i} \sigma_{j}=\sigma_{j} \sigma_{i} \text { if }|i-j| \geq 2\right\rangle .
$$

Consider the natural map $c: D \rightarrow S^{2}$ given by identifying $\partial D$, the boundary of $D$ to

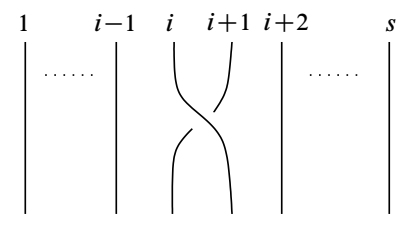

Figure 2: Braid generator $\sigma_{i}$

a point $p_{\infty}$ on $S^{2}$. By abuse of notation, we will write $\mathcal{S}$ for $c(\mathcal{S})$. Then there is an induced map:

$$
\begin{aligned}
\mathcal{B}(D ; \mathcal{S}) & \rightarrow \mathcal{B}\left(S^{2} ; \mathcal{S},\left\{p_{\infty}\right\}\right) \\
\beta & \mapsto \widehat{\beta} .
\end{aligned}
$$

For example, $\hat{\beta}_{m, n}$ and $\hat{\sigma}_{m, n}$ are shown in Figure 3 with the strand associated to $p_{\infty}$ drawn on the right. For $\beta \in \mathcal{B}(D ; \mathcal{S})$, let $\bar{\beta} \in \mathcal{B}\left(S^{2} ; \mathcal{S}\right)$ be the image of $\hat{\beta}$ under the 


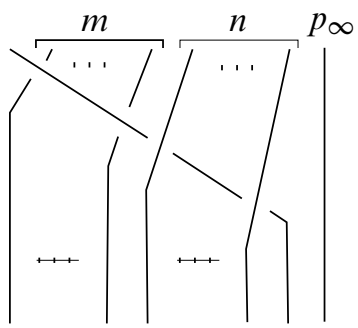

(a)

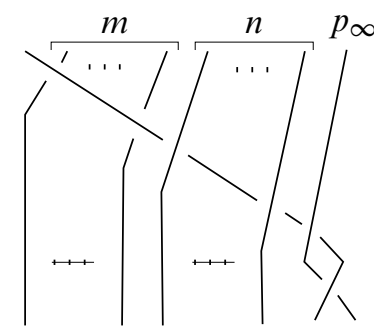

(b)

Figure 3: Images of (a) $\beta_{m, n}$ and (b) $\sigma_{m, n}$ in $\mathcal{B}\left(S^{2} ; \mathcal{S},\left\{p_{\infty}\right\}\right)$

forgetful map:

$$
\begin{aligned}
\mathcal{B}\left(S^{2} ; \mathcal{S},\left\{p_{\infty}\right\}\right) & \rightarrow \mathcal{B}\left(S^{2} ; \mathcal{S}\right) \\
\widehat{\beta} & \mapsto \bar{\beta}
\end{aligned}
$$

The following lemma can be found in the book by Birman [3].

Lemma 2.1 The map $\mathcal{B}(D ; \mathcal{S}) \rightarrow \mathcal{B}\left(S^{2} ; \mathcal{S}\right)$ given by composing the maps in (2) and (3) has kernel normally generated by $\xi=\sigma_{1} \sigma_{2} \ldots \sigma_{s-1}^{2} \sigma_{s-2} \ldots \sigma_{1}$.

For example, $\beta_{m, n}$ and $\sigma_{m, n}$ shown in Figure 1 differ by a conjugate of $\xi$, and hence we have the following.

Proposition 2.2 The braids $\beta_{m, n}$ and $\sigma_{m, n}$ satisfy $\bar{\beta}_{m, n}=\bar{\sigma}_{m, n}$.

The final lemma of this section deals with notation.

Lemma 2.3 Let $\mathcal{S}_{1}$ and $\mathcal{S}_{2}$ be finite subsets of $\operatorname{int}(F)$ with the same cardinality, and $h: F \rightarrow F$ any homeomorphism taking $\mathcal{S}_{1}$ to $\mathcal{S}_{2}$. Then conjugation by $h$ defines an isomorphism $\mathcal{B}\left(F ; \mathcal{S}_{1}\right) \rightarrow \mathcal{B}\left(F ; \mathcal{S}_{2}\right)$.

In light of Lemma 2.3 if $s$ is the cardinality of $\mathcal{S}$, we will write $\mathcal{B}(F, s)$ for $\mathcal{B}(F ; \mathcal{S})$.

\subsection{Mapping class groups}

For any closed orientable surface $F$ and a finite subset $\mathcal{S} \subset F$ of marked points, let $\mathcal{M}(F ; \mathcal{S})$ be the group of isotopy classes of orientation preserving homeomorphisms of $F$ set-wise preserving $\mathcal{S}$. The Thurston-Nielsen classification states that any homeomorphism of a surface is isotopic to one of three types, which we describe below. 
A map $\Phi: F \rightarrow F$ set-wise preserving $\mathcal{S}$ is defined to be periodic if some power of $\Phi$ equals the identity map; and reducible if there is a $\Phi$-invariant closed 1-submanifold whose complementary components in $F \backslash \mathcal{S}$ have negative Euler characteristic. A mapping class $\phi \in \mathcal{M}(F ; \mathcal{S})$ is periodic (respectively, reducible) if it contains a representative that is periodic (respectively, reducible).

Before defining the third type of mapping class, we will make some preliminary definitions. A singular foliation $\mathcal{F}$ on $F$ with respect to $\mathcal{S}$ is a partition of $F$ into a union of real intervals $(-\infty, \infty)$ and $[0, \infty)$ called leaves such that for each point $x \in F$, the foliation $\mathcal{F}$ near $x$ has one of the following types in a local chart around $x$ :

(F1) $x \in F$ is a regular point (we will also say a 2-pronged point) of $\mathcal{F}$ (Figure 4(a)).

(F2) $x \in F$ is an $n$-pronged singularity of $\mathcal{F}$ (Figure 4(b),(c)), where $n \geq 1$ if $x \in \mathcal{S}$, and $n \geq 3$ if $x \in F \backslash \mathcal{S}$.

Two singular foliations $\mathcal{F}^{+}$and $\mathcal{F}^{-}$with respect to $\mathcal{S}$ are transverse if they have the same set of singularities $\mathcal{S}^{\prime}$ and if the leaves of $\mathcal{F}^{+}$and $\mathcal{F}^{-}$intersect transversally on $F \backslash \mathcal{S}^{\prime}$

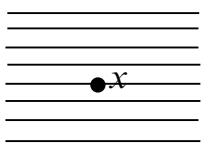

(a) $n=2$

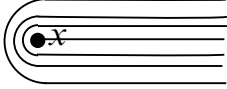

(b) $n=1$

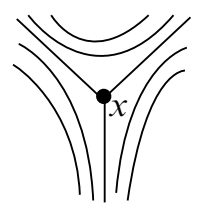

(c) $n=3$

Figure 4: Local picture of a singular foliation

A path $\alpha$ on $F$ is a transverse arc relative to a singular foliation $\mathcal{F}$ with respect to $\mathcal{S}$ if $\alpha$ intersects the leaves of $\mathcal{F}$ transversely. Two transverse $\operatorname{arcs} \alpha_{0}$ and $\alpha_{1}$ relative to $\mathcal{F}$ are homotopic if there is a homotopy $\alpha: I \times I \rightarrow F$ such that $\alpha(I \times 0)=\alpha_{0}$, $\alpha(I \times 1)=\alpha_{1}$, and for all $t \in I, \alpha(t \times I)$ is contained in a leaf of $\mathcal{F}$. We say that $\mu$ is a transverse measure on a singular foliation $\mathcal{F}$ with respect to $\mathcal{S}$ if $\mu$ defines a non-negative Borel measure $\mu(\alpha)$ on each transverse arc $\alpha$ with the following two properties:

(M1) If $\alpha^{\prime}$ is a subarc of $\alpha$, then $\mu\left(\alpha^{\prime}\right)=\left.\mu(\alpha)\right|_{\alpha^{\prime}}$.

(M2) If transverse $\operatorname{arcs} \alpha_{0}$ and $\alpha_{1}$ relative to $\mathcal{F}$ are homotopic, then $\mu\left(\alpha_{0}\right)=\mu\left(\alpha_{1}\right)$. 
A pair $(\mathcal{F}, \mu)$ satisfying (M1) and (M2) is called a measured foliation. Given a measured foliation $(\mathcal{F}, \mu)$ and a number $\lambda>0,(\mathcal{F}, \lambda \mu)$ denotes the measured foliation whose leaves are the same as those of $\mathcal{F}$ such that the measure of each transverse arc $\alpha$ relative to $\mathcal{F}$ is given by $\lambda \mu(\alpha)$. For a homeomorphism $f: F \rightarrow F$ set-wise preserving $\mathcal{S}$, $\left(\mathcal{F}^{\prime}, \mu^{\prime}\right)=f(\mathcal{F}, \mu)$ is the measured foliation whose leaves are the images of leaves of $\mathcal{F}$ under $f$, and the measure $\mu^{\prime}$ on each arc $\alpha$ transverse to $\mathcal{F}^{\prime}$ is given by $\mu\left(f^{-1}(\alpha)\right)$.

A map $\Phi: F \rightarrow F$ set-wise preserving $\mathcal{S}$ is pseudo-Anosov if there is a number $\lambda>1$ and a pair of transverse measured foliations $\left(\mathcal{F}^{ \pm}, \mu_{ \pm}\right)$such that $\Phi\left(\mathcal{F}^{ \pm}, \mu_{ \pm}\right)=$ $\left(\mathcal{F}^{ \pm}, \lambda^{ \pm 1} \mu_{ \pm}\right)$. The number $\lambda=\lambda(\Phi)$ is called the dilatation of $\Phi$, and $\mathcal{F}^{-}$and $\mathcal{F}^{+1}$ are called the stable and unstable foliations or the invariant foliations associated to $\Phi$. A mapping class $\phi \in \mathcal{M}(F ; \mathcal{S})$ is pseudo-Anosov if $\phi$ is the isotopy class of a pseudo-Anosov map $\Phi$. In this case, the dilatation of $\phi$ is defined to be $\lambda(\phi)=\lambda(\Phi)$.

Theorem 2.4 (Thurston-Nielsen Classification Theorem) Any element $\phi \in \mathcal{M}(F ; \mathcal{S})$ is either periodic, reducible or pseudo-Anosov. Furthermore, if $\phi$ is pseudo-Anosov, then the pseudo-Anosov representative of $\phi$ is unique up to conjugacy.

As with braids, for any partition $\mathcal{S}=\mathcal{S}_{1} \cup \cdots \cup \mathcal{S}_{r}$, there is a subgroup

$$
\mathcal{M}\left(F ; \mathcal{S}_{1}, \ldots, \mathcal{S}_{r}\right) \subset \mathcal{M}(F ; \mathcal{S})
$$

that preserves each $\mathcal{S}_{i}$ set-wise. There is a natural map

$$
\mathcal{M}\left(F ; \mathcal{S}_{1}, \ldots, \mathcal{S}_{r}\right) \rightarrow \mathcal{M}\left(F ; \mathcal{S}_{1}, \ldots, \mathcal{S}_{r-1}\right)
$$

called the forgetful map. For pseudo-Anosov mapping classes $\phi, \log (\lambda(\phi))$ can be interpreted as the minimal topological entropy among all representatives of $\phi$ (see Fathi-Laudenbach-Poenaru [10]). We thus have the following inequality on dilatations.

Lemma 2.5 Let $\phi \in \mathcal{M}\left(F ; \mathcal{S}_{1}, \ldots, \mathcal{S}_{r}\right)$, and $\psi \in \mathcal{M}\left(F ; \mathcal{S}_{1}, \ldots, \mathcal{S}_{r-1}\right)$ the image of $\phi$ under the forgetful map. If $\phi$ and $\psi$ are both pseudo-Anosov, then $\lambda(\phi) \geq \lambda(\psi)$.

Lemma 2.6 Let $\phi \in \mathcal{M}\left(F ; \mathcal{S}_{1}, \ldots, \mathcal{S}_{r}\right)$ be pseudo-Anosov. Suppose that the pseudoAnosov representative $\Phi$ of $\phi$ does not have a 1-pronged singularity at any point of $S_{r}$. Let $\psi \in \mathcal{M}\left(F ; \mathcal{S}_{1}, \ldots, \mathcal{S}_{r-1}\right)$ be the image of $\phi$ under the forgetful map. Then $\psi$ is pseudo-Anosov and $\lambda(\psi)=\lambda(\phi)=\lambda(\Phi)$.

Proof Let $\mathcal{F}^{ \pm}$be singular foliations with respect to $\mathcal{S}_{1} \cup \cdots \cup \mathcal{S}_{r}$, and $\left(\mathcal{F}^{ \pm}, \mu_{ \pm}\right)$ a pair of transverse measured foliations associated to $\Phi$. Since $\mathcal{F}^{ \pm}$does not have 1 -pronged singularities at points of $S_{r}, \mathcal{F}^{ \pm}$give well-defined singular foliations with respect to $\mathcal{S}_{1} \cup \cdots \cup \mathcal{S}_{r-1}$. Thus, $\Phi$ is a pseudo-Anosov representative of $\psi$, and hence $\lambda(\psi)=\lambda(\phi)=\lambda(\Phi)$. 
As in the case of braids, changing the location of the points in $\mathcal{S}$ by a homeomorphism does not change the group $\mathcal{M}(F ; \mathcal{S})$.

Lemma 2.7 Let $\mathcal{S}_{1}$ and $\mathcal{S}_{2}$ be two finite subsets of $F$ with the same cardinality, and $h: F \rightarrow F$ any homeomorphism taking $\mathcal{S}_{1}$ to $\mathcal{S}_{2}$. Then conjugation by $h$ defines an isomorphism $\mathcal{M}\left(F ; \mathcal{S}_{1}\right) \rightarrow \mathcal{M}\left(F ; \mathcal{S}_{2}\right)$.

If $F$ has genus $-g$, and $\mathcal{S}$ has cardinality $s$, we will also write $\mathcal{M}_{g}^{s}=\mathcal{M}(F ; \mathcal{S})$.

The theory of mapping class groups on closed surfaces extends to mapping class groups on surfaces with boundary. Let $F^{b}$ be a compact orientable surface with $b$ boundary components, and $\mathcal{S} \subset \operatorname{int}\left(F^{b}\right)$ a finite set. Define $\mathcal{M}\left(F^{b} ; \mathcal{S}\right)$ to be the group of isotopy classes of orientation preserving homeomorphisms of $F^{b}$ set-wise preserving $\mathcal{S}$ and the boundary components. A singular foliation $\mathcal{F}$ on $F^{b}$ with respect to the set of marked points $\mathcal{S}$ is a partition of $F$ into a union of leaves such that each point $x \in \operatorname{int}(F)$ has a local chart satisfying one of the conditions (F1), (F2), and each boundary component has $n$-prongs for some $n \geq 1$. Figure 5 illustrates representative leaves of a singular foliation with a 1-pronged (Figure 5(a)) and 3-pronged (Figure 5(b)) singularity. Periodic, reducible and pseudo-Anosov mapping classes are defined as for the case of closed surfaces using this definition of singular foliations.

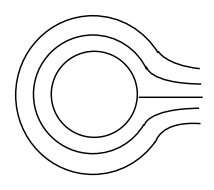

(a)

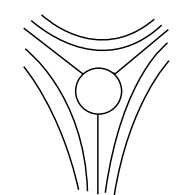

(b)

Figure 5: Leaves of a singular foliation near a boundary component

Let

$$
c: F^{b} \rightarrow \overline{F^{b}}
$$

be the continuous map, where $\overline{F^{b}}$ is the closed surface obtained from $F^{b}$ by contracting $b$ boundary components to points $q_{1}, \ldots, q_{b}$. As before, we will write $\mathcal{S}$ for $c(\mathcal{S})$. Let $\mathcal{Q}=\left\{q_{1}, \ldots, q_{b}\right\}$. The above definitions imply the following.

Lemma 2.8 The contraction map $c$ in (4) induces an isomorphism

$$
c_{*}: \mathcal{M}(F ; \mathcal{S}) \rightarrow \mathcal{M}\left(\overline{F^{b}} ; \mathcal{S}, \mathcal{Q}\right)
$$


which preserves the Thurston-Nielsen types of mapping classes. Furthermore, if $\mathcal{F}$ is a singular foliation defined on $F$ which is $n$-pronged along a boundary component $A$ of $F$, then the image of $\mathcal{F}$ under $c_{*}$ has an $n$-pronged singularity at $c_{*}(A)$.

The isomorphism $c_{*}$ given in Lemma 2.8 is handy in discussing mapping classes coming from braids. Let $F$ be either $D$ or $S^{2}$. There is a natural homomorphism

$$
\begin{aligned}
\mathcal{B}(F ; \mathcal{S}) & \rightarrow \mathcal{M}(F ; \mathcal{S}) \\
\beta & \mapsto \phi_{\beta}
\end{aligned}
$$

defined as follows. Let $D_{1}, \ldots, D_{s-1} \subset \operatorname{int}(D)$ be disks with $D_{i} \cap D_{j}=\varnothing$ for $i \neq j$ such that $D_{i}$ contains two points $p_{i}$ and $p_{i+1}$ of $\mathcal{S}$ and no other points of $\mathcal{S}$. The action of a generator $\sigma_{i}$ of $\mathcal{B}(F ; \mathcal{S})$ is the mapping class in $\mathcal{M}(F ; \mathcal{S})$ that fixes the exterior of $D_{i}$ and rotates a closed line segment connecting $p_{i}$ and $p_{i+1}$ in $D_{i}$ by 180 degrees in the counter-clockwise direction as in Figure 6.

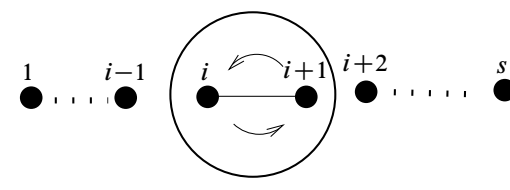

Figure 6: Action of $\sigma_{i}$ as a homeomorphism of $F$

Given a braid $\beta \in \mathcal{B}(D ; \mathcal{S})$, let $\widehat{\beta}$ be its image in $\mathcal{B}\left(S^{2} ; \mathcal{S},\left\{p_{\infty}\right\}\right)$ as in (2). Then $c_{*}$ satisfies $c_{*}\left(\phi_{\beta}\right)=\phi_{\widehat{\beta}}$.

The following useful lemma can be found in the book by Birman [3].

Lemma 2.9 If $\mathcal{S}$ has cardinality $s$, then the kernel of the map

$$
\begin{aligned}
\mathcal{B}(D ; \mathcal{S}) & \rightarrow \mathcal{M}\left(S^{2} ; \mathcal{S},\left\{p_{\infty}\right\}\right) \\
\beta & \mapsto \phi_{\widehat{\beta}}
\end{aligned}
$$

is the center $Z(\mathcal{B}(D ; \mathcal{S}))$ generated by a full twist braid $\Delta=\left(\sigma_{1} \ldots \sigma_{s-1}\right)^{s}$.

We say that $\beta \in \mathcal{B}(D ; \mathcal{S})$ is periodic (respectively, reducible, pseudo-Anosov), if $\phi_{\widehat{\beta}} \in \mathcal{M}\left(S^{2} ; \mathcal{S},\left\{p_{\infty}\right\}\right)$ is periodic (respectively, reducible, pseudo-Anosov). In the pseudo-Anosov case, we set $\lambda(\beta)=\lambda(\hat{\beta})=\lambda\left(\phi_{\widehat{\beta}}\right)$.

Let $\bar{\beta}$ be the image of $\hat{\beta}$ in $\mathcal{B}\left(S^{2} ; \mathcal{S}\right)$ under the forgetful map in (3). Then Lemma 2.5 implies that if $\hat{\beta}$ and $\bar{\beta}$ are pseudo-Anosov, we have $\lambda(\beta)=\lambda(\hat{\beta}) \geq \lambda(\bar{\beta})$, and by Lemma 2.6 , the equality holds if $p_{\infty}$ is not a 1 -pronged singularity for the invariant foliations associated to the pseudo-Anosov representative of $\phi_{\widehat{\beta}}$. 


\subsection{The braid spectrum}

For any subset $\Gamma \subset \mathcal{M}_{g}^{s}$, let $\Sigma(\Gamma)$ be the set of logarithms of dilatations coming from pseudo-Anosov elements of $\Gamma$. For any subset $\Gamma \subset \mathcal{B}(D, s)$, define $\Sigma(\Gamma)$ in a similar way. Let $\widehat{\mathcal{B}}(D, s) \subset \mathcal{M}_{0}^{s+1}$ be the image of $\mathcal{B}(D, s)$ under the map in Lemma 2.9, and $\widehat{\mathcal{B}}_{\mathrm{pA}}(D, s)$ the set of pseudo-Anosov elements of $\widehat{\mathcal{B}}(D, s)$.

Proposition 2.10 For $g \geq 1$,

$$
\Sigma(\mathcal{B}(D, 2 g+1))=\Sigma(\widehat{\mathcal{B}}(D, 2 g+1)) \subset \Sigma\left(\mathcal{M}_{g, \text { hyp }}\right) \subset \Sigma\left(\mathcal{M}_{g}\right) .
$$

Proof By using Lemma 2.9, it is easy to see that $\Sigma(\mathcal{B}(D, 2 g+1))=\Sigma(\widehat{\mathcal{B}}(D, 2 g+1))$.

Let $\mathcal{S} \subset \operatorname{int}(D)$ be a subset of $2 g+1$ points, and $\widehat{\mathcal{S}}=\mathcal{S} \cup\left\{p_{\infty}\right\}$. Let $F$ be the double cover of $S^{2}$ branched along $\widehat{\mathcal{S}}$. Then $F$ has genus $-g$. We will define a set map

$$
\widehat{\mathcal{B}}_{\mathrm{pA}}(D, 2 g+1) \rightarrow \mathcal{M}_{g}
$$

whose image consists of hyperelliptic elements which preserves dilatation. Let $\phi \in$ $\widehat{\mathcal{B}}_{\mathrm{pA}}(D, 2 g+1)$. Then $\phi$ has a pseudo-Anosov representative homeomorphism $\Phi$ that is unique up to conjugacy. Let $\Phi^{\prime}$ be its lift to $F$ by the covering $F \rightarrow S^{2}$ with invariant foliations given by the lifts of the invariant foliations associated to $\Phi$. Then $\Phi^{\prime}$ is pseudo-Anosov with the same dilatation as $\Phi$. Let $\phi^{\prime}$ be its isotopy class. Then $\phi^{\prime}$ defines a hyperelliptic, pseudo-Anosov mapping class in $\mathcal{M}\left(F ; \widehat{\mathcal{S}}^{\prime}\right)$ with the same dilatation as $\phi$, where $\widehat{\mathcal{S}}^{\prime}$ is the preimage of $\widehat{\mathcal{S}}$ in $F$.

Now consider the forgetful map $\mathcal{M}\left(F ; \widehat{\mathcal{S}}^{\prime}\right) \rightarrow \mathcal{M}(F ; \varnothing)=\mathcal{M}_{g}$. The invariant foliations associated to $\Phi^{\prime}$ have prong orders at $\widehat{\mathcal{S}}^{\prime}$ that are divisible by the degree of the covering $F \rightarrow S^{2}$. Thus, the singularities of $\Phi^{\prime}$ at $\widehat{\mathcal{S}}^{\prime}$ are all even-pronged. It follows that by Lemma 2.6, the image of $\phi^{\prime}$ under the forgetful map is pseudo-Anosov and has the same dilatations as $\phi^{\prime}$.

Proposition 2.10 immediately implies the following.

Corollary 2.11 For $g \geq 1, \delta\left(\mathcal{M}_{g}\right) \leq \delta\left(\mathcal{M}_{g \text {,hyp }}\right) \leq \delta(\mathcal{B}(D, 2 g+1))$.

\subsection{Criterion for the pseudo-Anosov property}

What follows is a criterion for determining when $\beta \in \mathcal{B}(D ; \mathcal{S})$ is pseudo-Anosov (see Bestvina-Handel [2]).

Let $G$ be a finite graph embedded on an orientable surface $F$, possibly with self-loops, but no vertices of valence 1 or 2 . Let $\mathcal{E}_{\operatorname{dir}}(G)$ be the set of oriented edges of $G, \mathcal{E}^{\text {tot }}(G)$ 
the set of unoriented edges, and $\mathcal{V}(G)$ the set of vertices. For $e \in \mathcal{E}_{\mathrm{dir}}(G)$, let $i(e)$ and $t(e)$ be the initial vertex and the terminal vertex respectively, and $\bar{e}$ the same edge with opposite orientation. An edge path $\tau$ on $G$ is an oriented path $\tau=e_{1} \ldots e_{\ell}$, where $e_{1}, \ldots, e_{\ell} \in \mathcal{E}_{\mathrm{dir}}(G)$ satisfies $t\left(e_{i}\right)=i\left(e_{i+1}\right)$ for $i=1, \ldots, \ell-1$. One can associate a fibered surface $\mathcal{F}(G) \subset F$ with a projection $\pi: \mathcal{F}(G) \rightarrow G$ (Figure 7). The fibered surface $\mathcal{F}(G)$ is decomposed into arcs and into polygons modelled on $k$-junctions for $k \geq 1$. The arcs and the $k$-junctions are called decomposition elements. Under $\pi$, the preimage of the vertices of valence $k$ of $G$ is the $k$-junctions, and the preimage of the edges of $G$ is the strips fibered by arcs, which are complementary components of the set of all junctions of $\mathcal{F}(G)$.

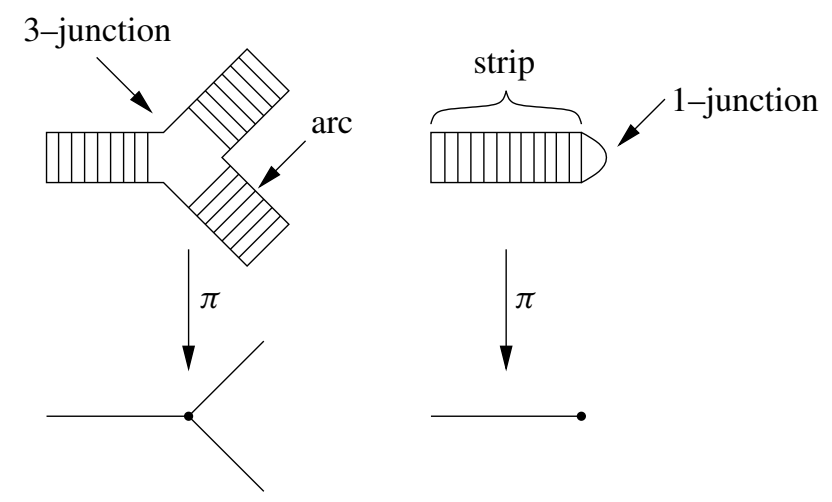

Figure 7: Fibered surface

Let $G$ and $H$ be finite graphs embedded on $F$, and $f: F \rightarrow F$ a homeomorphism. Assume that $f$ maps each decomposition element of $\mathcal{F}(G)$ into a decomposition element of $\mathcal{F}(H)$, and each junction of $\mathcal{F}(G)$ into a junction of $\mathcal{F}(H)$. Then $f$ induces a graph map $\mathfrak{g}: G \rightarrow H$ which sends vertices of $G$ to vertices of $H$, and each edge of $G$ to an edge path of $H$. Under this assumption with the case $G=H$, we say that $\mathcal{F}(G)$ carries $f$.

Let $V^{\text {tot }}(G)$ be the vector space of formal sums $\sum_{i=1}^{n} a_{i} e_{i}$, where $a_{i} \in \mathbb{R}$ and $e_{i} \in$ $\mathcal{E}^{\text {tot }}(G)$. Any edge path on $G$ determines an element of $V^{\text {tot }}(G)$ by treating each oriented edge as an unoriented edge with coefficient 1, regardless of orientation. For a graph map $\mathfrak{g}: G \rightarrow H$, define the transition matrix for $\mathfrak{g}$ to be the transformation

$$
\mathcal{T}_{\mathfrak{g}}^{\text {tot. }}: V^{\text {tot }}(G) \rightarrow V^{\text {tot }}(H)
$$

taking each $e \in \mathcal{E}^{\text {tot }}(G)$ to $\mathfrak{g}(e)$ considered as an element of $V^{\text {tot }}(H)$.

We now restrict to the case $F=D$. Let $\mathcal{S}=\left\{p_{1}, \ldots, p_{s}\right\} \subset \operatorname{int}(D)$ be a set of marked points, and $P_{i}$ a small circle centered at $p_{i}$ whose interior disk does not contain any 
other points of $\mathcal{S}$. We set $P=\bigcup_{i=1}^{S} P_{i}$. Choose a finite graph $G$ embedded on $D$ that is homotopy equivalent to $D \backslash \mathcal{S}$ such that $P$ is a subgraph of $G$. Given $\beta \in \mathcal{B}(D ; \mathcal{S})$, suppose that a fibered surface $\mathcal{F}(G)$ carries some homeomorphim $f$ of $\phi_{\beta} \in \mathcal{M}(D ; \mathcal{S})$. Then the graph map $\mathfrak{g}: G \rightarrow G$, called the induced graph map for $\phi_{\beta}$, preserves $P$ set-wise. Let pre $P$ be the set of edges $e \in \mathcal{E}^{\text {tot }}(G)$ such that $\mathfrak{g}^{k}(e)$ is contained in $P$ for some $k \geq 1$. By the definition of pre $P$, the transition matrix $\mathcal{T}_{\mathfrak{g}}^{\text {tot }}$ has the following form:

$$
\mathcal{T}_{\mathfrak{g}}^{\text {tot }}=\left(\begin{array}{ccc}
\mathcal{P} & \mathcal{A} & \mathcal{B} \\
0 & \mathcal{Z} & \mathcal{C} \\
0 & 0 & \mathcal{T}
\end{array}\right),
$$

where $\mathcal{P}$ and $\mathcal{Z}$ are the transition matrices associated to $P$ and pre $P$ respectively, and $\mathcal{T}$ is the transition matrix associated to the rest of edges $\mathcal{E}(G)$ called real edges. Let $V(G)$ be the subspace of $V^{\mathrm{tot}}(G)$ spanned by $\mathcal{E}(G)$. The matrix $\mathcal{T}$ is the restriction of $\mathcal{T}_{\mathfrak{g}}^{\text {tot }}$ to $V(G)$ and is called the transition matrix with respect to the real edges. The spectral radius of $\mathcal{T}$ is denoted by $\lambda(\mathcal{T})$.

Given a graph map $\mathfrak{g}: G \rightarrow G$, define the derivative $D_{\mathfrak{g}}: \mathcal{E}_{\mathrm{dir}}(G) \rightarrow \mathcal{E}_{\mathrm{dir}}(G)$ as follows: For $e \in \mathcal{E}_{\text {dir }}(G)$, write $\mathfrak{g}(e)=e_{1} e_{2} \ldots e_{\ell}$, where $e_{i} \in \mathcal{E}_{\text {dir }}(G)$. The image of $e$ under $D_{\mathfrak{g}}$ is defined by the initial edge $e_{1}$.

A graph map $\mathfrak{g}: G \rightarrow G$ is efficient if for any $e \in \mathcal{E}_{\text {dir }}(G)$ and any $k \geq 0, \mathfrak{g}^{k}(e)=$ $e_{1} e_{2} \ldots e_{j}$ satisfies $D_{\mathfrak{g}}\left(\bar{e}_{i}\right) \neq D_{\mathfrak{g}}\left(e_{i+1}\right)$ for all $i=1, \ldots, j-1$. We also say in this case that $\mathfrak{g}^{k}$ has no back track for any $k \geq 0$.

A nonnegative square matrix $M$ is irreducible if for every set of indices $i, j$, there is an integer $n_{i, j}>0$ such that the $(i, j)$ th entry of $M^{n_{i, j}}$ is strictly positive.

Theorem 2.12 (Bestvina-Handel [2]) Let $\beta \in \mathcal{B}(D ; \mathcal{S})$, and $\mathfrak{g}: G \rightarrow G$ the induced graph map for $\phi_{\beta}$. Suppose that

(BH1) $\mathfrak{g}$ is efficient, and

(BH2) the transition matrix $\mathcal{T}$ with respect to the real edges is irreducible with $\lambda(\mathcal{T})>1$.

Then $\beta$ is pseudo-Anosov with dilatation equal to $\lambda(\mathcal{T})$.

It is not hard to check that the criterion of Theorem 2.12 behaves well under conjugation of maps. For the case of braids, this yields the following.

Lemma 2.13 Let $\alpha_{1} \in \mathcal{B}(D ; \mathcal{S})$. Suppose that a fibered surface $\mathcal{F}(G)$ carries a homeomorphism $f \in \phi_{\alpha_{1}}$, and let $\mathfrak{g}_{1}: G \rightarrow G$ be the induced graph map for $\phi_{\alpha_{1}}$. We now consider a conjugate braid $\alpha_{2}$ with $\alpha_{2}=\gamma \alpha_{1} \gamma^{-1}$, and we take any homeomorphism 
$h \in \phi_{\gamma}$. Then a fibered surface $\mathcal{F}(h(G))$ carries a homeomorphism $h f h^{-1} \in \phi_{\alpha_{2}}$, and hence $h f h^{-1}$ induces a graph map $\mathfrak{g}_{2}: h(G) \rightarrow h(G)$, which is the induced graph map for $\phi_{\alpha_{2}}$. If $\mathfrak{g}_{1}$ satisfies (BH1) and (BH2), then $\mathfrak{g}_{2}$ also satisfies (BH1) and (BH2).

Let $\phi \in \mathcal{M}(D ; \mathcal{S})$ be pseudo-Anosov, and $\mathfrak{g}: G \rightarrow G$ the induced graph map for $\phi$ satisfying satisfying (BH1) and (BH2). We construct an associated train track obtained by graph smoothing given as follows: Let $\mathcal{E}_{v} \subset \mathcal{E}_{\text {dir }}(G)$ be the set of oriented edges of $G$ emanating from a vertex $v$. For $e_{1}, e_{2} \in \mathcal{E}_{v}, e_{1}$ and $e_{2}$ are equivalent if $D_{\mathfrak{g}}^{k}\left(e_{1}\right)=D_{\mathfrak{g}}^{k}\left(e_{2}\right)$ for some $k \geq 1$. A gate is an equivalence class in $\mathcal{E}_{v}$. The train track $\tau_{\mathfrak{g}}$ associated to $\mathfrak{g}$ is constructed using the following steps:

Step T1 Deform each pair of equivalent edges $e_{i}, e_{j} \in \mathcal{E}_{v}$ in a small neighborhood of $v$ so that $e_{i}$ and $e_{j}$ are tangent at $v$.

Step T2 Insert a small disk $N_{v}$ at each vertex $v$. For each gate $\gamma$, assign a point $p(\gamma)$ on the boundary of $N_{v}$.

Step T3 If, for some edge $e$ of $G$ and some $k \geq 1, \mathfrak{g}^{k}(e)$ contains consecutive edges $\bar{e}_{j} e_{\ell}\left(e_{j}, e_{\ell} \in \mathcal{E}_{v}\right)$ such that $\gamma_{j}=\left[e_{j}\right]$ and $\gamma_{\ell}=\left[e_{\ell}\right]$ with $\gamma_{j} \neq \gamma_{\ell}$, then join $p\left(\gamma_{j}\right)$ and $p\left(\gamma_{\ell}\right)$ by a smooth arc in $N_{v}$ satisfying the following: The arc intersects the boundary of $N_{v}$ transversally at $p\left(\gamma_{j}\right)$ and $p\left(\gamma_{\ell}\right)$, and no two such arcs intersect in the interior of $N_{v}$.

For example, let $v$ be the initial vertex of four edges $e_{1}, e_{2}, e_{3}, e_{4}$. Assume that there are three gates $\gamma_{1}=\left[e_{1}\right]=\left[e_{2}\right], \gamma_{2}=\left[e_{3}\right]$ and $\gamma_{3}=\left[e_{4}\right]$, and that there are edges $f_{1}$ and $f_{2}$ of $G$ such that $\mathfrak{g}^{r}\left(f_{1}\right)=\ldots \bar{e}_{2} e_{4} \ldots$ and $\mathfrak{g}^{s}\left(f_{2}\right)=\ldots \bar{e}_{3} e_{4} \ldots$ for some $r, s \geq 1$. Then Figure 8(a) shows Step T1 applied to $e_{1}$ and $e_{2}$, Figure 8(b) shows Step T2 applied to $\gamma_{1}, \gamma_{2}$ and $\gamma_{3}$, and Figure 8(c) shows Step T3, which yields arcs connecting $p\left(\gamma_{1}\right)$ to $p\left(\gamma_{3}\right)$, and $p\left(\gamma_{2}\right)$ to $p\left(\gamma_{3}\right)$.

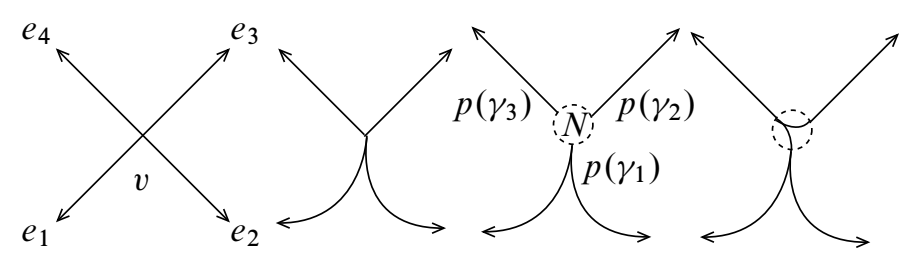

(a)

(b)

(c)

Figure 8: Example of a graph smoothing

The arcs constructed in Step T3 are called infinitesimal edges, and the points $p(\gamma)$ which join two infinitesimal edges are called cusps of the train track. 
If $\phi \in \mathcal{M}(D ; \mathcal{S})$ is pseudo-Anosov, and $\mathfrak{g}: G \rightarrow G$ is the induced graph map for $\phi$ satisfying (BH1) and (BH2), then $\tau_{\mathfrak{g}}$ constructed above determines the invariant foliations $\mathcal{F}^{ \pm}$associated to the pseudo-Anosov representative $\Phi$ of $\phi$. In particular, the number of prongs at the singularities of $\mathcal{F}^{ \pm}$can be found in terms of $\tau_{\mathfrak{g}}$. Each connected component $A$ of $D \backslash \tau_{\mathfrak{g}}$ is either homeomorphic to an open disk, or is a half-open annulus, one of whose boundaries is the boundary $\partial D$ of $D$. In the former case, the boundary of the closure of the connected component is a finite union of edges and vertices of $\tau_{\mathfrak{g}}$. If two of these edges meet at a cusp, then that cusp is said to belong to $A$. In the latter case, the closure of $A$ has two boundary components. The boundary component which is not $\partial D$ is a finite union of edges and vertices of $\tau_{\mathfrak{g}}$, and if two of these edges meet at a cusp, we call the cusp an exterior cusp of $\tau_{\mathfrak{g}}$.

Lemma 2.14 Let $A$ be a connected component of $D \backslash \tau_{\mathfrak{g}}$. If $A$ is an open disk, then there is one $k$-pronged singularity of $\mathcal{F}^{ \pm}$in $A$, where $k$ is the number of cusps of $\tau_{\mathfrak{g}}$ belonging to $A$. If $A$ is a half-open annulus, then $\partial D$ is $k$-pronged, where $k$ is the number of exterior cusps of $\tau_{\mathfrak{g}}$.

\section{Main examples}

This section contains properties of $\beta_{m, n}$ and $\sigma_{m, n}$. In Section 3.1, we show that the Thurston-Nielsen types of $\beta_{m, n}$ and $\sigma_{m, n}$ do not depend on the order of $m$ and $n$. In Section 3.2, we find the Thurston-Nielsen types of $\beta_{m, n}$ and $\sigma_{m, n}$, and in Section 3.4, we compute their dilatations in the pseudo-Anosov cases. Section 3.3 gives the

train tracks for $\phi_{\beta_{m, n}}$ and $\phi_{\sigma_{m, n}}$ In Section 3.5, we apply properties of Salem-Boyd sequences to find the least dilatation among $\lambda\left(\sigma_{m, n}\right)$ and $\lambda\left(\beta_{m, n}\right)$ for $m+n=2 g$ fixing $g \geq 2$. We also give bounds on these dilatations.

\subsection{Symmetries of $\beta_{m, n}$ and $\sigma_{m, n}$}

Consider the braid $\beta_{m, n}^{+} \in \mathcal{B}(D ; \mathcal{S},\{p\},\{q\})$ drawn in Figure 9(a).

Lemma 3.1 The braid $\beta_{n, m}^{+}$is conjugate to the inverse of $\beta_{m, n}^{+}$.

Proof The inverse of $\beta_{m, n}^{+}$is drawn in Figure 9(b). Assume without loss of generality that the points of $\mathcal{S} \cup\{p\} \cup\{q\}$ are evenly spaced along a line $\ell$. Let $\eta \in \mathcal{B}(D ; \mathcal{S} \cup\{p, q\})$ be the braid obtained by a half-twist of $\ell$ around the barycenter of $\mathcal{S} \cup\{p\} \cup\{q\}$. Then conjugating the inverse of $\beta_{m, n}^{+}$by $\eta$ in $\mathcal{B}(D ; \mathcal{S} \cup\{p, q\})$ yields $\beta_{n, m}^{+}$shown in Figure 9(c). 


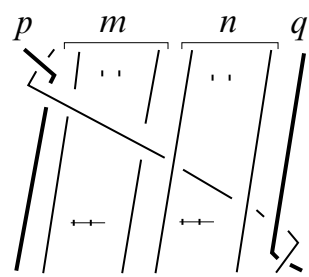

(a)

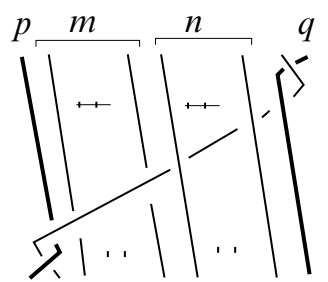

(b)

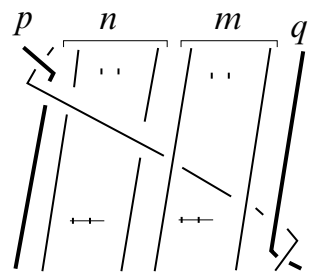

(c)

Figure 9: Symmetry of $\beta_{m, n}^{+}$

Lemma 3.2 The braid $\beta_{m, n}$ is the image of $\beta_{m, n}^{+}$under the forgetful map

$$
\mathcal{B}(D ; \mathcal{S},\{p\},\{q\}) \rightarrow \mathcal{B}(D ; \mathcal{S}),
$$

and hence $\beta_{n, m}$ is conjugate to $\beta_{m, n}^{-1}$.

Proof Compare Figure 3(a) with Figure 9(a) to get the first part of the claim. Since homomorphisms preserve inverses and conjugates, the rest follows from Lemma 3.1. $\square$

Lemma 3.2 together with the homomorphism in (5) shows the following.

Lemma 3.3 The mapping class $\phi_{\beta_{n, m}}$ is conjugate to $\phi_{\beta_{m, n}}^{-1}$.

Proposition 3.4 The Thurston-Nielsen type of $\beta_{n, m}$ is the same as that of $\beta_{m, n}$.

Proof The Thurston-Nielsen type of a mapping class is preserved under inverses and conjugates. Thus, the claim follows from Lemma 2.8 and Lemma 3.3.

We now turn to $\sigma_{m, n}$. Let $\hat{\beta}_{m, n}^{+}$and $v$ be the spherical braids drawn in Figures 10(a) and 11 respectively.

Lemma 3.5 The spherical braid $\widehat{\sigma}_{m, n}$ is the image of $v \hat{\beta}_{m, n}^{+} v^{-1}$ under the forgetful $\operatorname{map} \mathcal{B}\left(S^{2} ; \mathcal{S},\{p\},\{q\},\left\{p_{\infty}\right\}\right) \rightarrow \mathcal{B}\left(S^{2} ; \mathcal{S},\left\{p_{\infty}\right\}\right)$, and hence $\widehat{\sigma}_{n, m}$ is conjugate to $\hat{\sigma}_{m, n}^{-1}$.

Proof Compare Figure 10(a) and Figure 10(b) to get the first part of the claim. The rest follows by using the same argument in the proof of Lemma 3.2.

Remark 3.6 In the statement of Lemma 3.5, $v$ could be replaced by any braid which is the identity on $p$ and $\mathcal{S}$, and interchanges $q$ and $p_{\infty}$. 


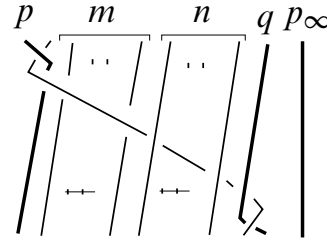

(a)

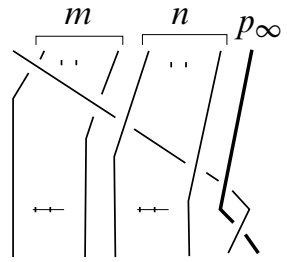

(b)

Figure 10: Spherical braids (a) $\hat{\beta}_{m, n}^{+}$and (b) $\hat{\sigma}_{m, n}$

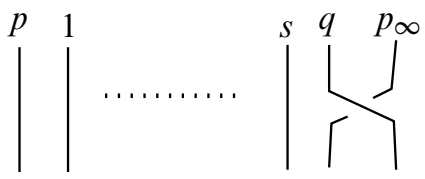

Figure 11: Spherical braid $v$ : Switching the roles of $p_{\infty}$ and $q$

Lemma 3.7 The mapping class $\phi_{\sigma_{n, m}}$ is conjugate to $\phi_{\sigma_{m, n}}^{-1}$.

Proof By Lemma 3.5, $\phi_{\widehat{\sigma}_{n, m}}$ is conjugate to $\phi_{\widehat{\sigma}_{m, n}}^{-1}$. Since the contraction map $c$ in (4) induces the isomorphism $c_{*}$ on mapping class groups, the claim follows.

Lemma 3.7 immediately shows the following.

Proposition 3.8 The Thurston-Nielsen type of $\sigma_{m, n}$ is the same as that for $\sigma_{n, m}$.

\subsection{Graph maps}

Theorem 3.9 The braid $\beta_{m, n}$ is pseudo-Anosov for all $m, n \geq 1$, and $\lambda\left(\beta_{m, n}\right)=$ $\lambda\left(\beta_{n, m}\right)$.

Let $G_{m, n}$ be the graph with vertices $1, \ldots, m+n+1, p$ and $q$ in Figure 12(left). Consider the graph map $\mathfrak{g}=\mathfrak{g}_{m, n}: G_{m, n} \rightarrow G_{m, n}$ given in Figure 12, where the ordering of the loop edges of $G_{m, n}$ corresponds to the left-to-right ordering of $\beta_{m, n}$. We denote the oriented edge with the initial vertex $a$ and the terminal vertex $b$ by $e(a, b)$.

Proposition 3.10 The graph map $\mathfrak{g}_{m, n}: G_{m, n} \rightarrow G_{m, n}$ is the induced graph map for $\phi_{\beta_{m, n}}$ satisfying (BH1) and (BH2). 

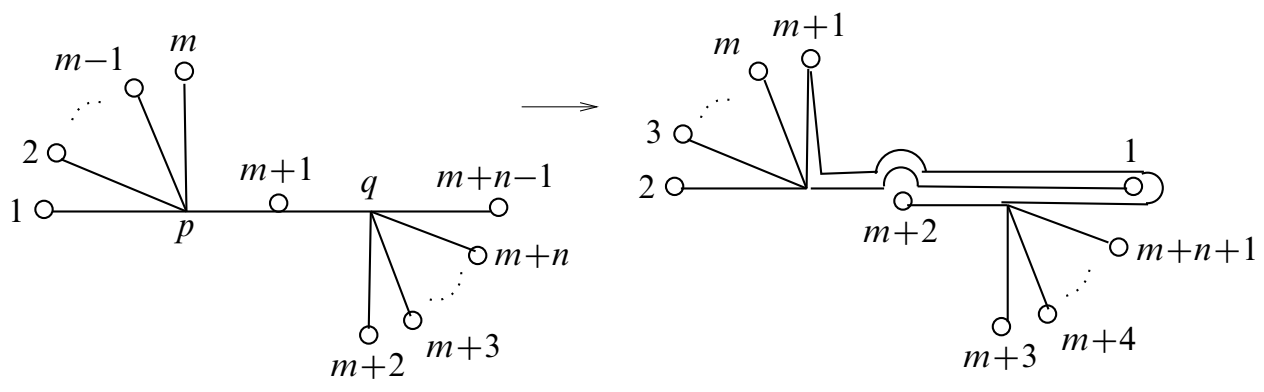

Figure 12: Graph map $\mathfrak{g}_{m, n}$ for $\phi_{\beta_{m, n}}$

Proof It is easy to see that the fibered surface $\mathcal{F}\left(G_{m, n}\right)$ carries a homeomorphism of $\phi_{\beta_{m, n}}$, and hence $\mathfrak{g}_{m, n}: G_{m, n} \rightarrow G_{m, n}$ is the induced graph map for $\phi_{\beta_{m, n}}$.

As shown in Figure 12, any back track must occur at $e(p, m)$, that is, if $\mathfrak{g}^{k}$ has back tracks, and $k$ is chosen minimally, then there is an edge $e \in \mathcal{E}_{\mathrm{dir}}\left(G_{m, n}\right)$ such that

$$
\mathfrak{g}^{k-1}(e)=\ldots \bar{e}_{1} \cdot e_{2} \ldots \text { with } D_{\mathfrak{g}}\left(e_{1}\right)=D_{\mathfrak{g}}\left(e_{2}\right)=e(m, p) .
$$

This implies that $\bar{e}_{1}=e(p, m+1)$ and $e_{2}=e(m+1, q)$ (or $\bar{e}_{1}=e(q, m+1)$ and $\left.e_{2}=e(m+1, p)\right)$. As can be seen by Figure 12, one can verify that there can be no edge of the form given in (6). This proves (BH1).

To prove (BH2), it suffices to note that $\mathfrak{g}^{m+n}(e(q, m+1))$ crosses all non-loop edges of $G_{m, n}$ in either direction, and for any non-loop edge $e$ of $G_{m, n}, \mathfrak{g}^{k}(e)$ crosses $e(q, m+1)$ in either direction for some $k \geq 1$.

Proof of Theorem 3.9 By Proposition 3.10, $\beta_{m, n}$ is pseudo-Anosov for all $m, n \geq 1$. By Lemma 3.3, we have $\lambda\left(\beta_{m, n}\right)=\lambda\left(\beta_{n, m}\right)$.

We now turn to $\sigma_{m, n}$.

Theorem 3.11 The braid $\sigma_{m, n}$ is pseudo-Anosov for all $m, n \geq 1$ satisfying $|m-n| \geq$ 2 . In these cases $\lambda\left(\sigma_{m, n}\right)=\lambda\left(\sigma_{n, m}\right)$. For any $m \geq 1, \sigma_{m, m}$ is periodic, and $\sigma_{m, m+1}$ and $\sigma_{m+1, m}$ are reducible.

In light of Proposition 3.8, we will consider only $\sigma_{m, n}$ when $n \geq m \geq 1$. To prove Theorem 3.11, we first redraw $\sigma_{m, n}$ in a conjugate form using induction. Let $\tau_{a, b}$ be the spherical braid drawn in Figure 13. Roughly speaking, conjugation by $\tau_{a, b}$ on $\sigma_{m, n}$ is the same as passing a strand counterclockwise around the other strands, and then compensating below after a shift of indices. 


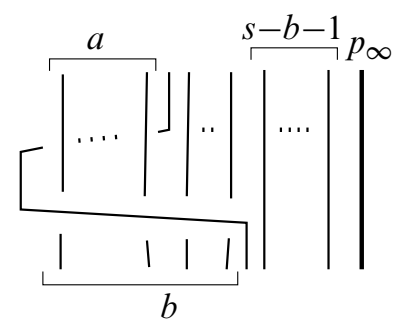

Figure 13: Spherical braid $\tau_{a, b} \in \mathcal{B}\left(S^{2} ; \mathcal{S},\left\{p_{\infty}\right\}\right)$
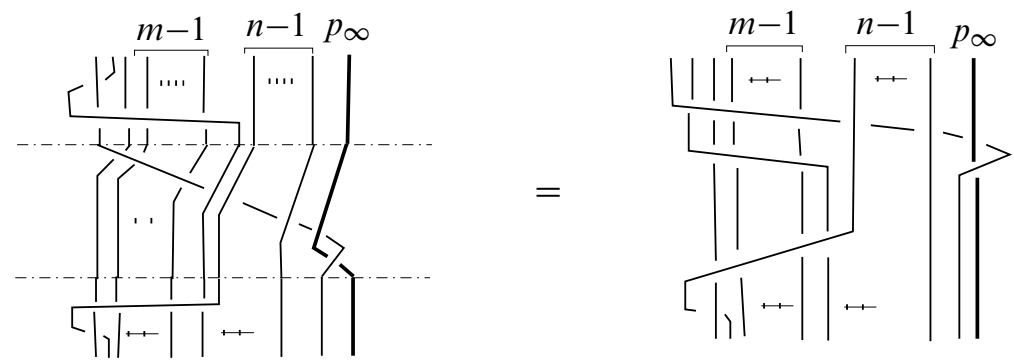

Figure 14: Conjugating $\widehat{\sigma}_{m, n}$ : Initial step

Let $\widehat{\sigma}_{m, n}^{(0)}=\widehat{\sigma}_{m, n}$ be the image of $\sigma_{m, n}$ in $\mathcal{B}\left(S^{2} ; \mathcal{S},\left\{p_{\infty}\right\}\right)$ as drawn in Figure 10(b). Let

$$
\hat{\sigma}_{m, n}^{(1)}=\tau_{1, m+1} \hat{\sigma}_{m, n} \tau_{1, m+1}^{-1}
$$

shown in Figure 14. The inductive step is illustrated in Figure 15. The $k$ th braid $\hat{\sigma}_{m, n}^{(k)}$ is constructed from the $(k-1)$ st braid by conjugating by $\tau_{2 k+1, m+k+1}$ for $k=1, \ldots, m-1$. The resulting braid $\hat{\sigma}_{m, n}^{(m-1)}$ takes one of three forms: Figure 16(a) shows the general case when $n \geq m+2$, Figure 16(b) shows the case when $n=m+1$, and Figure 16(c) shows the case when $n=m$.

Proposition 3.12 When $n=m+1, \sigma_{m, n}$ is a reducible braid.

Proof By applying one more conjugation by $\tau_{2 m+1,2 m+1}$, we obtain the left-hand braid in Figure 17(a), which equals the right-hand braid. One sees that there is a collection of disjoint disks enclosing pairs of marked points in $S^{2}$ whose boundaries are invariant by $\phi_{\widehat{\sigma}_{m, n}}$. The claim now follows from Lemma 2.8 .

Proposition 3.13 When $n=m, \sigma_{m, n}$ is a periodic braid. 


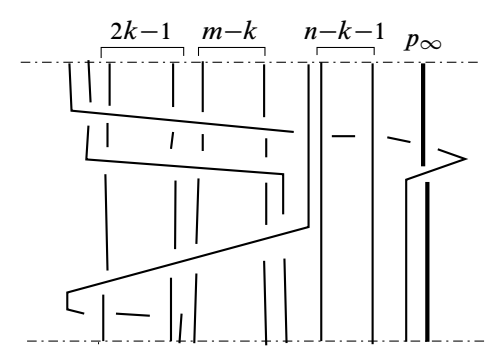

(a)

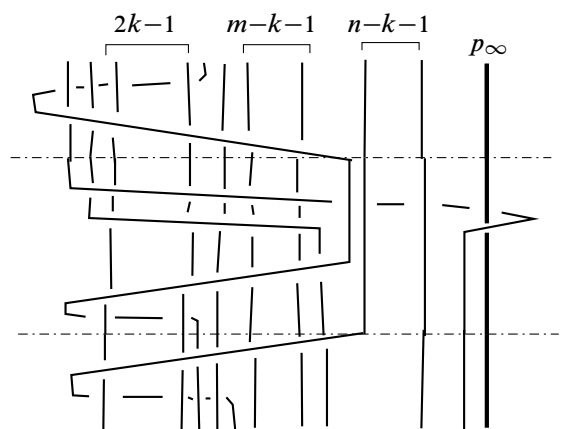

(b)

Figure 15: Induction step

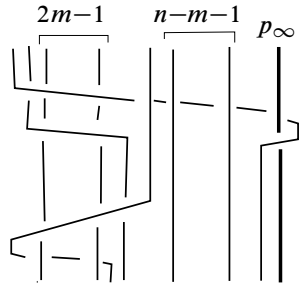

(a)

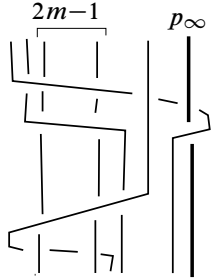

(b)

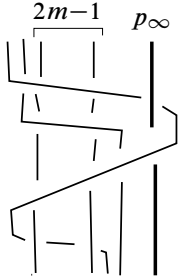

(c)

Figure 16: After $(m-1)$ inductive steps: (a) $n \geq m+2$ (b) $n=m+1$ (c) $n=m$

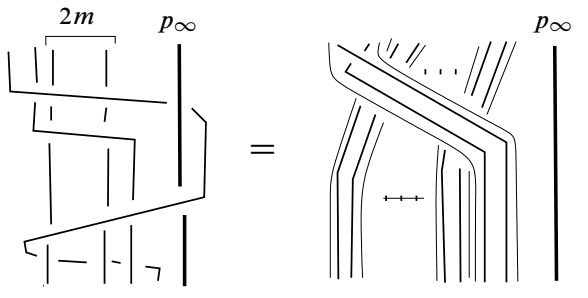

(a)

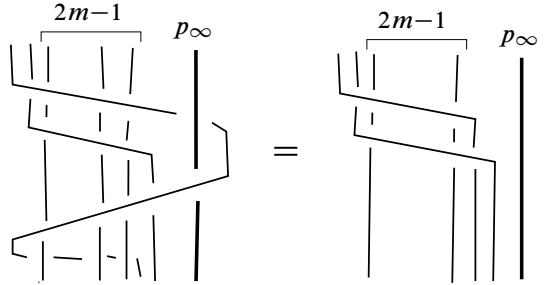

(b)

Figure 17: (a) reducible and (b) periodic cases

Proof Figure 17(b) shows an equivalence of spherical braids. It is not hard to see that the right-hand braid is periodic in $\mathcal{B}\left(S^{2} ; \mathcal{S},\left\{p_{\infty}\right\}\right)$. The rest follows from Lemma 2.8 .

Algebraic 83 Geometric Topology, Volume 6 (2006) 
The general case when $n \geq m+2$ is shown in Figure 18. The transition from Figure 18(a) to 18 (b) is given by doing successive conjugations by $\tau_{2 m+k, 2 m+k}$ for $k=1, \ldots, n-m$. The braid in 18(b) equals the braid in 18(c).

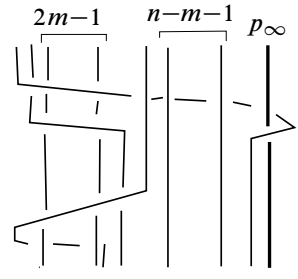

(a)
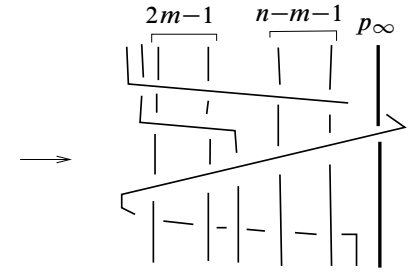

(b)

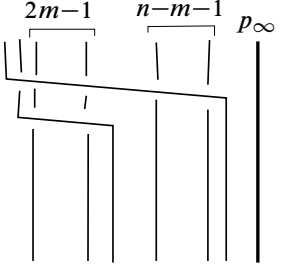

(c)

Figure 18: General case

Let $H_{m, n}$ be the graph with vertices $1, \ldots, m+n+1$ and $p$ in Figure 19(left), and we consider the graph map $\mathfrak{h}_{m, n}: H_{m, n} \rightarrow H_{m, n}$ drawn in Figure 19. The unusual numbering of vertices comes from the left-to-right ordering of the strands (excluding $\left.p_{\infty}\right)$ of $\hat{\sigma}_{m, n}$ shown in Figure 3(b). This ordering proves useful for comparing the transition matrices of $\phi_{\beta_{m, n}}$ and $\phi_{\sigma_{m, n}}$ in Section 3.5.

Let $\sigma_{m, n}^{\prime} \in \mathcal{B}(D ; \mathcal{S})$ be the braid given by the preimage of the braid in Figure 18(c) under the contraction map of Lemma 2.8. (Hence $\sigma_{m, n}$ is obtained from the braid in Figure $18(\mathrm{c})$ by removing the strand $p_{\infty}$.)
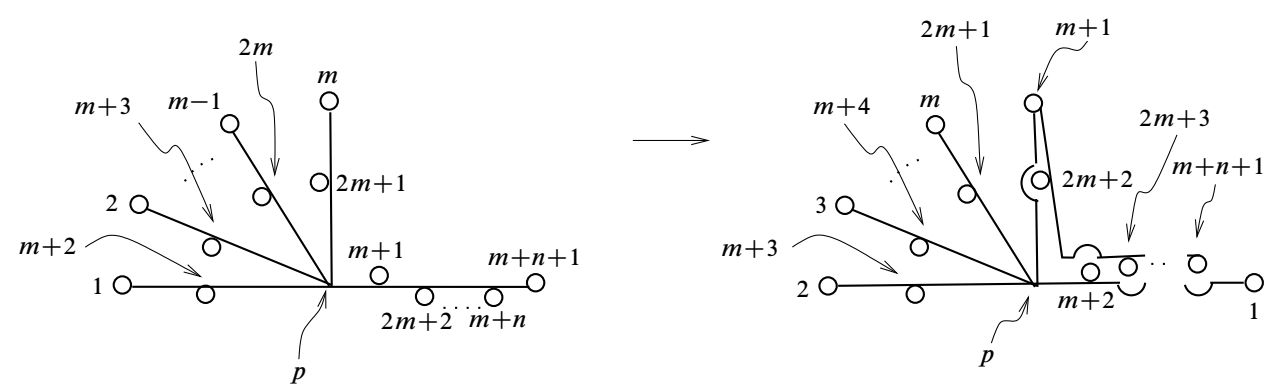

Figure 19: Graph map $\mathfrak{h}_{m, n}$ for $\phi_{\sigma_{m, n}}$

Proposition 3.14 For $n \geq m+2$, the graph map $\mathfrak{h}_{m, n}: H_{m, n} \rightarrow H_{m, n}$ is the induced graph map for $\phi_{\sigma_{m, n}^{\prime}}$ satisfying (BH1) and (BH2). 
Proof One can see that the fibered surface $\mathcal{F}\left(H_{m, n}\right)$ carries a homeomorphism of $\phi_{\sigma_{m, n}^{\prime}}$, and hence $\mathfrak{h}_{m, n}: H_{m, n} \rightarrow H_{m, n}$ is the induced graph map for $\phi_{\sigma_{m, n}^{\prime}}$. The proof that $\mathfrak{h}_{m, n}$ satisfies (BH1) and (BH2) is similar to that of Proposition 3.10.

Proof of Theorem 3.11 By Proposition 3.8, it suffices to classify the braids $\sigma_{m, n}$ with $n \geq m \geq 1$. By Proposition 3.12, $\sigma_{m, n}$ is reducible if $n=m+1$, and by Proposition $3.13 \sigma_{m, n}$ is periodic if $n=m$. In all other cases, Proposition 3.14 shows that $\sigma_{m, n}$ is pseudo-Anosov since $\sigma_{m, n}$ is conjugate to $\sigma_{m, n}^{\prime}$, and Lemma 3.7 implies that $\lambda\left(\sigma_{m, n}\right)=\lambda\left(\sigma_{n, m}\right)$.

\subsection{Train tracks}

By using the graph smoothing in Section 2.4, the train track $\tau_{\mathfrak{g}_{m, n}}$ for $\phi_{\beta_{m, n}}$ and the train track $\tau_{\mathfrak{h}_{m, n}}$ for $\phi_{\sigma_{m, n}^{\prime}}$ are given in Figures 20 and 21. Applying Lemma 2.14 to $\tau_{\mathfrak{g} m, n}$ and $\tau_{\mathfrak{h}_{m, n}}$, we immediately see the following.

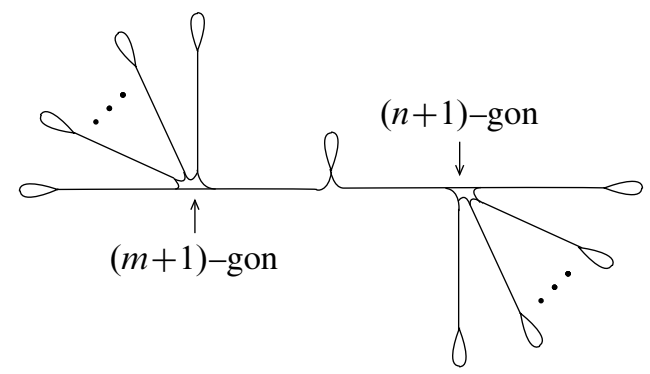

Figure 20: Train track $\tau_{\mathfrak{g} m, n}$

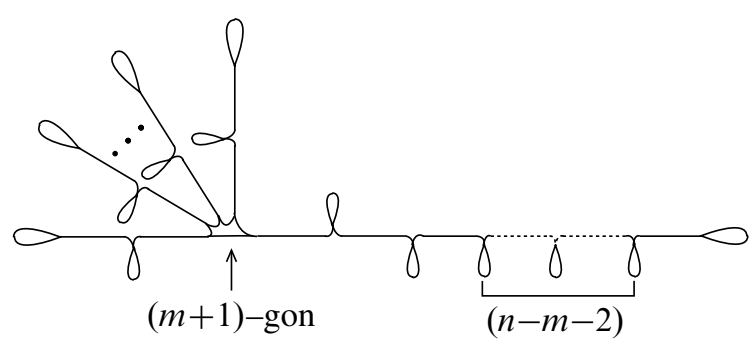

Figure 21: Train track $\tau_{\mathfrak{h} m, n}$

Algebraic 83 Geometric Topology, Volume 6 (2006) 
Lemma 3.15 The invariant foliations associated to the pseudo-Anosov representative $\Phi_{\widehat{\beta}_{m, n}}$ of $\phi_{\widehat{\beta}_{m, n}} \in \mathcal{M}\left(S^{2} ; \mathcal{S},\left\{p_{\infty}\right\}\right)$ have a 1 -pronged singularity at each point of $\mathcal{S} \cup\left\{p_{\infty}\right\}$, an $(m+1)$-pronged singularity at a point $p \in S^{2} \backslash\left(\mathcal{S} \cup\left\{p_{\infty}\right\}\right)$, and an $(n+1)$-pronged singularly at a point $q \in S^{2} \backslash\left(\mathcal{S} \cup\left\{p_{\infty}\right\}\right)$.

Lemma 3.16 For $n \geq m+2$, the invariant foliations associated to the pseudo-Anosov representative $\Phi_{\widehat{\sigma}_{m, n}}$ of $\phi_{\widehat{\sigma}_{m, n}} \in \mathcal{M}\left(S^{2} ; \mathcal{S},\left\{p_{\infty}\right\}\right)$ have a 1 -pronged singularity at each point of $\mathcal{S}$, an $n$-pronged singularity at $p_{\infty}$, and an $(m+1)$-pronged singularity at a point $p \in S^{2} \backslash\left(\mathcal{S} \cup\left\{p_{\infty}\right\}\right)$.

By Lemmas 2.6 and 3.16, we have the following.

Corollary 3.17 For $n \geq m+2, \bar{\sigma}_{m, n}$ is pseudo-Anosov, and $\lambda\left(\sigma_{m, n}\right)=\lambda\left(\bar{\sigma}_{m, n}\right)$.

A pseudo-Anosov map $\Phi$ is said to be orientable if the stable and unstable foliations associated to $\Phi$ are orientable.

Proposition 3.18 Let $m+n=2 g$. If both $m$ and $n$ are odd, there is a pseudoAnosov element of $\mathcal{M}_{g}$ whose pseudo-Anosov representative is orientable with the same dilatation as $\beta_{m, n}$.

Proof Let $\Phi \widehat{\beta}_{m, n}$ be the lift of $\Phi \widehat{\beta}_{m, n}$ to the double branched covering $F_{g}$ of $S^{2}$ branched along $\mathcal{S} \cup\left\{p_{\infty}\right\}$, and denote by $\widehat{\mathcal{S}}^{\prime}$ the preimage of $\widehat{\mathcal{S}}=\mathcal{S} \cup\left\{p_{\infty}\right\}$ in $F_{g}$. By the proof of Proposition 2.10, $\Phi_{\widehat{\beta}_{m, n}}$ is a pseudo-Anosov map with $\lambda\left(\Phi_{\hat{\beta}_{m, n}}\right)=$ $\lambda\left(\Phi \widehat{\beta}_{m, n}\right)=\lambda\left(\beta_{m, n}\right)$. By Lemma 3.15, $\Phi_{\widehat{\beta}_{m, n}}$ has an invariant foliation $\mathcal{F}^{ \pm}$with two $(m+1)$-pronged singularities and two $(n+1)$-pronged singularities at points of $F_{g} \backslash \widehat{\mathcal{S}}^{\prime}$, and regular points of $\widehat{\mathcal{S}}^{\prime}$. Hence all singularities of $\mathcal{F}^{ \pm}$are even-pronged.

To show that $\mathcal{F}^{ \pm}$is orientable, it suffices to note that the natural map from the fundamental group of $F_{g}$ to $Z / 2 Z$ induced by $\mathcal{F}^{ \pm}$is trivial. Consider the invariant foliation on $S^{2}$ associated to $\Phi \widehat{\beta}_{m, n}$ with 1 -pronged singularity at each point of $\widehat{\mathcal{S}}$ and even-pronged singularity elsewhere. The punctured sphere $S^{2} \backslash \widehat{\mathcal{S}}$ has fundamental group generated by loops emanating from a basepoint, following a path $\gamma_{p}$ to a point near a marked point $p \in \widehat{\mathcal{S}}$, going around a small circle centered at $p$, then returning in the reverse direction along $\gamma_{p}$ back to the basepoint. Consider the double unbranched covering of $S^{2} \backslash \widehat{\mathcal{S}}$. Then by construction, the natural map from the fundamental group of the covering surface to $Z / 2 Z$ defined by the lifted foliation is trivial. The same is true for the fundamental group of the branched covering surface $F_{g}$, and hence the natural map from the fundamental group of $F_{g}$ to $Z / 2 Z$ defined by the lifted foliation $\mathcal{F}^{ \pm}$is trivial. 
Proposition 3.19 Let $m+n=2 g$. For each $m, n \geq 1$ with $|m-n| \geq 2$, there is a pseudo-Anosov element of $\mathcal{M}_{g}$ whose pseudo-Anosov representative is orientable with the same dilatation as $\sigma_{m, n}$.

Proof By Lemma 3.7, we can assume $n \geq m+2$. Lemma 3.16 says that the invariant foliations associated to $\Phi_{\widehat{\sigma}_{m, n}}$ have an $n$-pronged singularity and an $(m+1)$-pronged singularity. Since $m+n=2 g,(m+1)$ and $n$ have the opposite parity.

Let $F_{g}$ be the branched covering of $S^{2}$ branched along $\mathcal{S}$ and either an $(m+1)-$ pronged singularity if $(m+1)$ is odd, or $p_{\infty}$ if $n$ is odd. Let $\Phi_{\widehat{\sigma}_{m, n}}^{\prime}$ be the lift of $\Phi_{\widehat{\sigma}_{m, n}}$ to $F_{g}$. Then $\Phi_{\widehat{\sigma}_{m, n}}^{\prime}$ is pseudo-Anosov with dilatation equal that of $\Phi_{\widehat{\sigma}_{m, n}}$. Furthermore, by our choice of branch points, the invariant foliations have only even order prongs. One shows that they are orientable by using the same arguments as in the proof of Proposition 3.18.

We conclude this section by relating $\mathfrak{g}_{m, n}$ and $\mathfrak{h}_{m, n}$ in a way that is compatible with the conjugations used in Section 3.1.

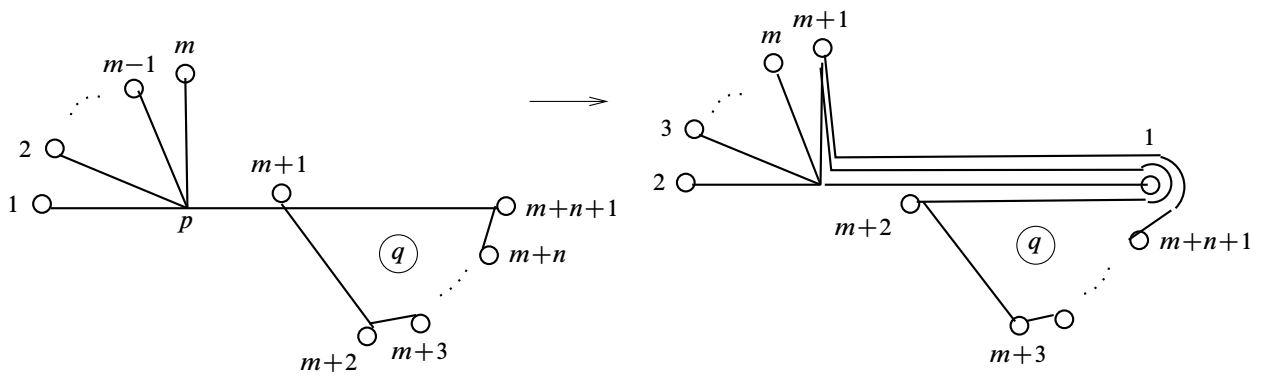

Figure 22: Graph map $\mathfrak{g}_{m, n}^{\prime}$ for $\phi_{m, n}$

Since $q$ is a fixed point for $\mathfrak{g}_{m, n}$ (see Figure 12), $\Phi_{\widehat{\beta}_{m, n}}$ determines a mapping class $\phi_{m, n}=\left[\Phi_{\widehat{\beta}_{m, n}}\right]$ in $\mathcal{M}\left(S^{2} ; \mathcal{S},\{q\},\left\{p_{\infty}\right\}\right)$. Let $\mathfrak{g}_{m, n}^{\prime}:{ }_{G_{m, n}^{\prime n}}^{\prime n} \rightarrow G_{m, n}^{\prime}$ be the graph map obtained from $\mathfrak{g}_{m, n}$ after puncturing $D$ at $q$ as in Figure 22. Then $\mathfrak{g}_{m, n}^{\prime}: G_{m, n}^{\prime} \rightarrow G_{m, n}^{\prime}$ is the induced graph (satisfying (BH1) and (BH2)) for the mapping class which is the preimage of $\phi_{m, n}$ under the map from $\mathcal{M}(D ; \mathcal{S},\{q\})$ to $\mathcal{M}\left(S^{2} ; \mathcal{S},\{q\},\left\{p_{\infty}\right\}\right)$. Identify $\mathfrak{g}_{m, n}^{\prime}$ with the graph map on $S^{2}$ obtained by pushed forward by the contraction map in Lemma 2.8.

Exchanging the roles of $q$ and $p_{\infty}$ (i.e., bringing $p_{\infty}$ into the visual plane) yields the graph map shown in Figure 23, which is equivalent to $\mathfrak{g}_{m, n}^{\prime}$. Now remove $p_{\infty}$, and consider the graph map

$$
\mathfrak{f}_{m, n}: G_{m, n}^{\prime} \rightarrow H_{m, n}
$$

Algebraic 83 Geometric Topology, Volume 6 (2006) 

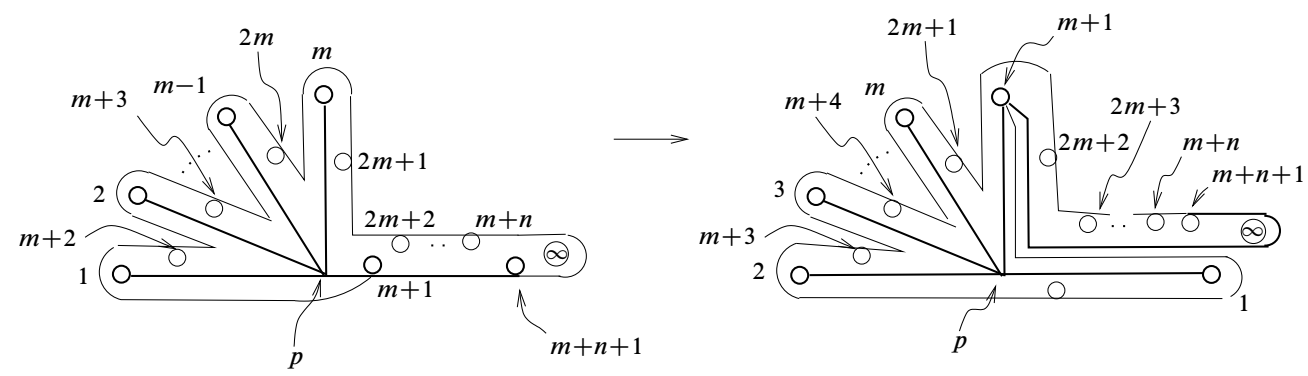

Figure 23: Exchanging the roles of $q$ and $p_{\infty}$ for $\mathfrak{g}_{m, n}^{\prime}: \infty$ in the figure indicates $p_{\infty}$

obtained by a natural identification of edges of $G_{m, n}^{\prime}$ to edges of the graph in Figure 22(left) removing $p_{\infty}$. Figure 24 shows the natural projection map applied to the image of the edges of $G_{m, n}^{\prime}$ under $\mathfrak{f}_{m, n}$. The map $\mathfrak{h}_{m, n}: H_{m, n} \rightarrow H_{m, n}$ in Figure 19 is the one induced by pushing forward $\mathfrak{g}_{m, n}^{\prime}$ by the map $\mathfrak{f}_{m, n}$.
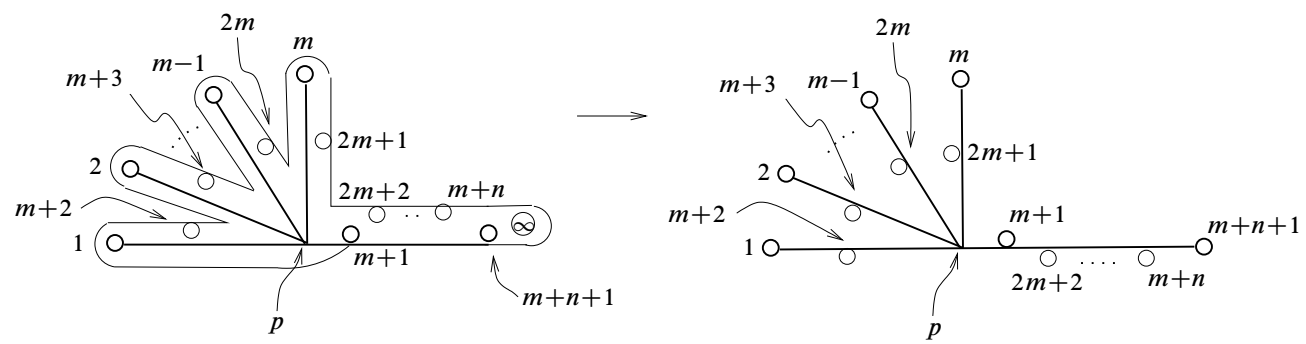

Figure 24: Identifying edges of $G_{m, n}^{\prime}$ with edges of $H_{m, n}$

\subsection{Characteristic equations for dilatation}

Consider the graph map $\mathfrak{r}_{m}: \Gamma_{m} \rightarrow \Gamma_{m}$, shown in Figure 25 . As seen in Figures 12 and 19, the graph maps for $\phi_{\beta_{m, n}}$ and $\phi_{\sigma_{m, n}}$ "contain" $\mathfrak{r}_{m}$ as the action on a subgraph. 

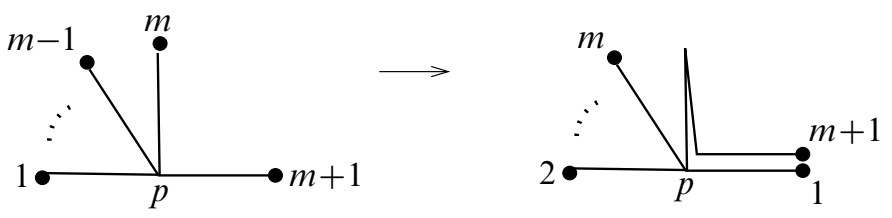

Figure 25: Graph map $\mathfrak{r}_{m}: \Gamma_{m} \rightarrow \Gamma_{m}$

The transition matrix for $\mathfrak{r}_{m}$ has the following form with respect to the basis of edges $e(p, 1), \ldots, e(p, m+1)$ :

$$
\mathcal{R}_{m}=\left[\begin{array}{cccccc}
0 & 1 & 0 & \ldots & 0 & 0 \\
0 & 0 & 1 & \ldots & 0 & 0 \\
\vdots & & & & & \\
0 & 0 & 0 & \ldots & 1 & 0 \\
0 & 0 & 0 & \ldots & 0 & 2 \\
1 & 0 & 0 & \ldots & 0 & 1
\end{array}\right]
$$

The characteristic polynomial for $\mathcal{R}_{m}$ is $R_{m}(t)=t^{m}(t-1)-2$. As we will see in the proof of Theorem 3.20, the appearance of $\mathcal{R}_{m}$ within the transition matrices of $\phi_{\beta_{m, n}}$ and $\phi_{\sigma_{m, n}}$ gives rise to a similar form for their characteristic equations.

Given a polynomial $f(t)$ of degree $d$, the reciprocal of $f(t)$ is $f_{*}(t)=t^{d} f(1 / t)$.

Theorem 3.20 (1) For $m, n \geq 1, \lambda\left(\beta_{m, n}\right)$ is the largest root of

$$
T_{m, n}(t)=t^{n+1} R_{m}(t)+\left(R_{m}\right)_{*}(t) .
$$

(2) For $m, n \geq 1$ with $|m-n| \geq 2, \lambda\left(\sigma_{m, n}\right)$ is the largest root of

$$
S_{m, n}(t)=t^{n+1} R_{m}(t)-\left(R_{m}\right)_{*}(t) .
$$

Proof We note that the spectral radius of the transition matrix for $\mathfrak{g}_{m, n}$ is equal to that for $\mathfrak{g}_{m, n}^{\prime}$ by the construction of $\mathfrak{g}_{m, n}^{\prime}$ (Section 3.3). Thus, to find characteristic polynomials for $\lambda\left(\beta_{m, n}\right)$ and $\lambda\left(\sigma_{m, n}\right)$, it is enough to compute the transition matrices for $\mathfrak{g}_{m, n}^{\prime}$ and $\mathfrak{h}_{m, n}$ respectively.

Consider the basis for $V^{\text {tot }}\left(G_{m, n}^{\prime}\right)$ :

$$
\begin{aligned}
v_{k} & =e(p, k), \quad k=1, \ldots, m, \\
v_{m+1} & =e(p, m+1)+e(m+1, m+n+1), \\
v_{m+1+k} & =e(m+k, m+k+1), \quad k=1, \ldots, n, \text { and } \\
v_{m+n+2} & =e(p, m+1) .
\end{aligned}
$$

Algebraic $8 \mathcal{G}$ Geometric Topology, Volume 6 (2006) 
The corresponding transition matrix $\mathcal{T}_{m, n}^{\prime}$ for $\mathfrak{g}_{m, n}^{\prime}$ is given by:

$$
\mathcal{T}_{m, n}^{\prime}=\left[\begin{array}{cccccc|ccccc}
0 & 1 & 0 & \ldots & 0 & 0 & 0 & 0 & \ldots & & 0 \\
0 & 0 & 1 & \ldots & 0 & 0 & 0 & 0 & \ldots & & 0 \\
& & & \ldots & & & & & \ldots & & \\
0 & 0 & 0 & \ldots & 1 & 0 & 0 & 0 & \ldots & & 0 \\
0 & 0 & 0 & \ldots & 0 & 2 & 1 & 0 & \ldots & & (1)_{b} \\
1 & 0 & 0 & \ldots & 0 & 1 & 2 & 0 & \ldots & & 0 \\
\hline 0 & 0 & \ldots & & 0 & 0 & 0 & 1 & 0 & \ldots & 0 \\
0 & 0 & \ldots & & 0 & 0 & 0 & 0 & 1 & \ldots & 0 \\
& & \ldots & & & & & \ldots & & \\
0 & 0 & \ldots & & 0 & 0 & 0 & 0 & \ldots & 1 & 0 \\
0 & 0 & \ldots & & 0 & (1)_{a b} & 0 & 0 & \ldots & 0 & 0 \\
0 & 0 & \ldots & & 0 & 0 & (-1)_{b} & 0 & \ldots & 0 & (0)_{a}
\end{array}\right]
$$

We will show that the characteristic polynomial for $\mathcal{T}_{m, n}^{\prime}$ is given by

$$
T_{m, n}(t)=t^{n+1} R_{m}(t)+\left(R_{m}\right)_{*}(t) .
$$

The upper left block matrix of $\mathcal{T}_{m, n}^{\prime}$ corresponding to the vectors $v_{1}, \ldots, v_{m+1}$ is identical to $\mathcal{R}_{m}$. Multiplying the characteristic polynomials of the upper left and lower right diagonal blocks gives $t^{n+1} R_{m}$. The rest of the characteristic polynomial has two nonzero summands. One corresponds to the matrix entries marked $a$, and is given by

$$
t(-1)^{n+1}\left|\begin{array}{ccccc}
-1 & 0 & \cdots & 0 & 0 \\
t & -1 & \cdots & 0 & 0 \\
& & \cdots & & \\
0 & 0 & \cdots & t & -1
\end{array}\right|_{(n-1) \times(n-1)}\left|\begin{array}{ccccc}
t & -1 & \cdots & 0 & 0 \\
0 & t & \cdots & 0 & 0 \\
& & \cdots & & \\
0 & 0 & \cdots & t & -1 \\
-1 & 0 & \cdots & 0 & -2
\end{array}\right|_{(m+1) \times(m+1)}
$$

which yields $-t\left(2 t^{m}+1\right)$. The other summand corresponds to the matrix entries marked $b$ and is given by

$$
(-1)^{n+1}\left|\begin{array}{ccccc}
-1 & 0 & \cdots & 0 & 0 \\
t & -1 & \cdots & 0 & 0 \\
& & \cdots & & \\
0 & 0 & \cdots & t & -1
\end{array}\right|_{(n-2) \times(n-2)}\left|\begin{array}{cccccc}
t & -1 & 0 & \cdots & 0 & 0 \\
0 & t & -1 & \cdots & 0 & 0 \\
& & & \cdots & & \\
0 & 0 & 0 & \cdots & t & -1 \\
-1 & 0 & 0 & \cdots & 0 & 0
\end{array}\right|_{m \times m}
$$

which yields 1 . This completes the proof of (1).

Let $\mathcal{S}_{m, n}$ be the transition matrix for $\mathfrak{h}_{m, n}: H_{m, n} \rightarrow H_{m, n}$. We will pull back $\mathcal{S}_{m, n}$ to an invertible linear transformation on $V^{\text {tot }}\left(G_{m, n}^{\prime}\right)$ using $\mathfrak{f}_{m, n}$ given in (7). Let 
$\mathfrak{h}_{m, n}^{\prime}\left(v_{i}\right)$ be the image of $\mathfrak{g}_{m, n}^{\prime}\left(v_{i}\right)$ under $\mathfrak{f}_{m, n}$. Then the transition matrix $\mathcal{S}_{m, n}^{\prime}$ for $\mathfrak{h}_{m, n}^{\prime}: G_{m, n}^{\prime} \rightarrow H_{m, n}$ is the same as $\mathcal{T}_{m, n}^{\prime}$ except at the vector $v_{m+n+1}$. As can be seen in Figure 23, we have

$$
\mathcal{S}_{m, n}^{\prime}\left(v_{m+1}\right)=\mathcal{T}_{m, n}^{\prime}\left(v_{m+1}\right)-2 v_{m+n+1}
$$

Thus, $\mathcal{S}_{m, n}^{\prime}$ differs from $\mathcal{T}_{m, n}^{\prime}$ only by changing the entry labeled by both $a$ and $b$ from 1 to -1 .

Recall that the sign of the entry marked both $a$ and $b$ in $\mathcal{T}_{m, n}^{\prime}$ determines the sign of in front of $\left(R_{m}\right)_{*}$. Since this sign is the only difference between $\mathcal{S}_{m, n}^{\prime}$ and $\mathcal{T}_{m, n}^{\prime}$, the characteristic polynomial for $\mathcal{S}_{m, n}^{\prime}$ is given by

$$
S_{m, n}(t)=t^{n+1} R_{m}(t)-\left(R_{m}\right)_{*}(t) .
$$

To finish the proof of (2), we have left to check that $\lambda\left(\sigma_{m, n}\right)$ is the largest root of $S_{m, n}$. Thus (2) follows if we can show that the extra eigenvalue of $\mathcal{S}_{m, n}^{\prime}$ has absolute value 1. From Figure 24, we see that the kernel of the linear map induced by $\mathfrak{f}_{m, n}$ is spanned by

$$
w=2\left(v_{1}+\cdots+v_{m}\right)+v_{m+1}-\left(v_{m+2}+\cdots+v_{m+n+1}\right)+v_{m+n+2} .
$$

Under $\mathfrak{h}_{m, n}^{\prime}$, we have

$$
\begin{aligned}
2\left(v_{1}+\cdots v_{m}\right) & \mapsto 2\left(v_{m+1}+v_{1}+\cdots+v_{m-1}\right), \\
v_{m+1} & \mapsto 2 v_{m}+v_{m+1}-v_{m+n+1}, \\
v_{m+2}+\cdots+v_{m+n+1} & \mapsto v_{m}+2 v_{m+1}+v_{m+2}+\cdots+v_{m+n}-v_{m+n+2}, \\
v_{m+n+2} & \mapsto v_{m},
\end{aligned}
$$

and hence, $\mathfrak{h}_{m, n}^{\prime}(w)=w$. Thus, the characteristic polynomial for $\mathcal{S}_{m, n}^{\prime}$ differs from that for $\mathcal{S}_{m, n}$ by a factor of $(t-1)$.

Remark 3.21 Minakawa independently discovered the pseudo-Anosov maps on $F_{g}$ constructed in the proof of Proposition 3.19 for the case when $(m, n)=(g-1, g+1)$ using a beautiful new method for constructing orientable pseudo-Anosov maps on $F_{g}$ (see Minakawa [24]). He also directly computes their dilatation using different techniques from ours.

\subsection{Dilatations and Salem-Boyd sequences}

Recall that given a polynomial $f(t)$ of degree $d$, the reciprocal of $f(t)$ is $f_{*}(t)=$ $t^{d} f(1 / t)$. The polynomial $f$ satisfying $f=f_{*}$ (respectively, $f=-f_{*}$ ) is a reciprocal 
polynomial (respectively, anti-reciprocal polynomial). For a monic integer polynomial $P(t)$ of degree $d$, the sequence

$$
Q_{n}^{ \pm}(t)=t^{n} P(t) \pm P_{*}(t)
$$

is called the Salem-Boyd sequence associated to $P$.

Theorem 3.22 Let $Q_{n}$ be a Salem-Boyd sequence associated to $P$. Then $Q_{n}$ is a reciprocal or an anti-reciprocal polynomial, and the set of roots of $Q_{n}$ outside the unit circle converge to those of $P$ as $n$ goes to infinity.

Theorem 3.22 is a consequence of Rouché's Theorem applied to the sum $\frac{P(t)}{t^{d}} \pm \frac{P_{*}(t)}{t^{n+d}}$ considered as a holomorphic function on the Riemann sphere minus the unit disk.

For a monic integer polynomial $f(t)$, let $N(f)$ be the number of roots of $f$ outside the unit circle, $\lambda(f)$ the maximum norm of roots of $f$, and $M(f)$ the product of the norms of roots outside the unit circle, which is called the Mahler measure of $f$. By Theorem 3.22, we have the following.

Corollary 3.23 Let $Q_{n}$ be a Salem-Boyd sequence associated to $P$. Then

$$
\lim _{n \rightarrow \infty} M\left(Q_{n}\right)=M(P) \text { and } \lim _{n \rightarrow \infty} \lambda\left(Q_{n}\right)=\lambda(P) .
$$

Any algebraic integer on the unit circle has a (anti-)reciprocal minimal polynomial. Suppose that $P(t)=P_{0}(t) R(t)$, where $R$ is a (anti-)reciprocal and $P_{0}$ has no roots on the unit circle. Then the Salem-Boyd sequence associated to $P$ satisfies

$$
Q_{n}(t)=R(t)\left(t^{n} P_{0}(t) \pm\left(P_{0}\right)_{*}(t)\right) .
$$

We have thus shown the following.

Lemma 3.24 All roots of $P$ on the unit circle are also roots of $Q_{n}$ for all $n$.

The following theorem can be proved by first restricting to the case when $P$ has no roots on the unit circle, and then by defining a natural deformation of the roots of $P(t)$ to those of $Q_{n}(t)$, which don't cross the unit circle (see Boyd [4]).

Theorem 3.25 Let $Q_{n}$ be a Salem-Boyd sequence associated to $P$. Then $N\left(Q_{n}\right) \leq$ $N(P)$ for all $n$

We now apply the above results to the Salem-Boyd sequences $S_{m, n}$ and $T_{m, n}$ associated to $R_{m}$ of Theorem 3.20. To do this, we first study $R_{m}$. 
Lemma 3.26 For all $m \geq 1, M\left(R_{m}\right)=2$.

Proof For $|t|<1$, we have $\left|t^{m}(t-1)\right|<2$, and hence $R_{m}$ has no roots strictly within the unit circle. Therefore, the Mahler measure of $R_{m}$ must equal the absolute value of the constant coefficient, namely 2 .

Applying Corollary 3.23, we have the following.

Corollary 3.27 Fixing $m \geq 1$ and letting $n$ increase, the Mahler measures of $T_{m, n}$ and $S_{m, n}$ converge to 2 .

Lemma 3.28 The polynomial $R_{m}$ has one real root outside the unit circle. This root is simple and greater than 1.

Proof Taking the derivative $R_{m}^{\prime}(t)=(m+1) t^{m}-m t^{m-1}$, we see that $R_{m}$ is increasing for $t>\frac{m}{m+1}$, and hence also for $t \geq 1$. Since $R_{m}(1)=-2<0$ and $R_{m}(2)>0$, it follows that $R_{m}$ has a simple root $\mu_{m}$ with $1<\mu_{m}<2$. Similarly, we can show that for $t<0, R_{m}$ has no roots for $m$ even, and one root if $m$ is odd. In the odd case, $R_{m}(-1)=0$, so $R_{m}$ has no real roots strictly less than -1 .

Lemma 3.29 The sequence $\lambda\left(R_{m}\right)$ converges monotonically to 1 from above.

Proof Since $M\left(R_{m}\right)=2$, we know that $\mu_{m}=\lambda\left(R_{m}\right)>1$. Take any $\epsilon>0$. Let $D_{\epsilon}$ be the disk of radius $1+\epsilon$ around the origin in the complex plane. Let $g(t)=\frac{t-1}{t}$ and $h_{m}(t)=\frac{-2}{t^{m+1}}$. Then for large enough $m$, we have

$$
|g(t)|=\left|\frac{t-1}{t}\right|>\left|\frac{2}{t^{m+1}}\right|=\left|h_{m}(t)\right|
$$

for all $t$ on the boundary of $D_{\epsilon}$, and $g(t)$ and $h_{m}(t)$ are holomorphic on the complement of $D_{\epsilon}$ in the Riemann sphere. By Rouché's theorem, $g(t), g(t)+h_{m}(t)$, and hence $R_{m}(t)$ have the same number of roots outside $D_{\epsilon}$, which is zero.

To show the monotonicity consider $R_{m}\left(\mu_{m+1}\right)$. Note that $\left(\mu_{m+1}\right)^{m+1}(t-1)-2=0$. Hence we have

$$
\begin{aligned}
R_{m}\left(\mu_{m+1}\right) & =\left(\mu_{m+1}\right)^{m}(t-1)-2 \\
& =\left(\left(\mu_{m+1}\right)^{m}-\left(\mu_{m+1}\right)^{m+1}\right)(t-1) \\
& <0 .
\end{aligned}
$$

Since $R_{m}(t)$ is an increasing function for $t>1$, we conclude that $\mu_{m+1}<\mu_{m}$. 
Corollary 3.23 and Lemma 3.29 imply the following.

Corollary 3.30 Fixing $m \geq 1$, the sequences $\lambda\left(\beta_{m, n}\right)$ and $\lambda\left(\sigma_{m, n}\right)$ converge to $\lambda\left(R_{m}\right)$ as sequences in $n$. Furthermore, we can make $\lambda\left(\beta_{m, n}\right)$ and $\lambda\left(\sigma_{m, n}\right)$ arbitrarily close to 1 by taking $m$ and $n$ large enough.

We now determine the monotonicity of $\lambda\left(\beta_{m, n}\right)$ and $\lambda\left(\sigma_{m, n}\right)$ for fixed $m \geq 1$.

Proposition 3.31 Fixing $m \geq 1$, the dilatations $\lambda\left(\beta_{m, n}\right)$ are strictly monotone decreasing, and the dilatations $\lambda\left(\sigma_{m, n}\right)$ are strictly monotone increasing for $n \geq m+2$.

Proof Consider $f(t)=\left(R_{m}\right)_{*}(t)=-2 t^{m+1}-t+1$. Then, for $t>0$,

$$
f^{\prime}(t)=-2(m+1) t^{m}-1<0 .
$$

Also $f(1)=-2<0$. Since $b_{m, n}=\lambda\left(\beta_{m, n}\right)>1$, and for $n \geq m+2, s_{m, n}=\lambda\left(\sigma_{m, n}\right)>1$, it follows that $\left(R_{m}\right)_{*}\left(b_{m, n}\right)$ and $\left(R_{m}\right)_{*}\left(s_{m, n}\right)$ are both negative. We have

$$
\begin{aligned}
& 0=T_{m, n}\left(b_{m, n}\right)=\left(b_{m, n}\right)^{n+1} R_{m}\left(b_{m, n}\right)+\left(R_{m}\right)_{*}\left(b_{m, n}\right), \text { and } \\
& 0=S_{m, n}\left(s_{m, n}\right)=\left(s_{m, n}\right)^{n+1} R_{m}\left(s_{m, n}\right)-\left(R_{m}\right)_{*}\left(s_{m, n}\right),
\end{aligned}
$$

which imply that $R_{m}\left(b_{m, n}\right)>0$ and $R_{m}\left(s_{m, n}\right)<0$. Since $R_{m}$ is increasing for $t>1$, we have

$$
s_{m, n}<\mu_{m}<b_{m, n} .
$$

Plug $b_{m, n}$ into $T_{m, n-1}$, and subtract $T_{m, n}\left(b_{m, n}\right)=0$ :

$$
\begin{aligned}
T_{m, n-1}\left(b_{m, n}\right) & =\left(b_{m, n}\right)^{n-1} R_{m}\left(b_{m, n}\right)+\left(R_{m}\right)_{*}\left(b_{m, n}\right) \\
& =\left(\left(b_{m, n}\right)^{n-1}-\left(b_{m, n}\right)^{n}\right) R_{m}\left(b_{m, n}\right) \\
& <0
\end{aligned}
$$

Since $b_{m, n-1}$ is the largest real root of $T_{m, n-1}$, we have $b_{m, n}<b_{m, n-1}$.

We can show that $s_{m, n}<s_{m, n+1}$ for $n \geq m+2$ in a similar way, by adding the formula for $S_{m, n}\left(s_{m, n}\right)$ to $S_{m, n+1}\left(s_{m, n}\right)$.

The inequalities (8) give the following.

Corollary 3.32 For all $m, n \geq 1$ with $|m-n| \geq 2, \lambda\left(\beta_{m, n}\right)>\lambda\left(\sigma_{m, n}\right)$.

We now fix $2 g=m+n(g \geq 2)$, and show that among the braids $\beta_{m, n}$ and $\sigma_{m, n}$, $\sigma_{g-1, g+1}$ has the least dilatation. 
Proposition 3.33 (1) For $k=1, \ldots, m-1$,

$$
\begin{aligned}
\lambda\left(\beta_{m, m}\right) & <\lambda\left(\beta_{m-k, m+k}\right), \text { and } \\
\lambda\left(\beta_{m, m+1}\right) & <\lambda\left(\beta_{m-k, m+k+1}\right) .
\end{aligned}
$$

(2) For $k=2, \ldots, m-1$,

$$
\begin{aligned}
& \lambda\left(\sigma_{m-1, m+1}\right)<\lambda\left(\sigma_{m-k, m+k}\right), \text { and } \\
& \lambda\left(\sigma_{m-1, m+2}\right)<\lambda\left(\sigma_{m-k, m+k+1}\right) .
\end{aligned}
$$

Proof Let $\lambda=\lambda\left(\beta_{m, m}\right)$. Then plugging $\lambda$ into $T_{m-k, m+k}$ gives

$$
\begin{aligned}
T_{m-k, m+k}(\lambda) & =\lambda^{m+k+1}\left(\lambda^{m-k}(\lambda-1)-2\right)-2 \lambda^{m-k+1}-\lambda+1 \\
& =\lambda^{2 m+2}-\lambda^{2 m+1}-2 \lambda^{m+k+1}-2 \lambda^{m-k+1}-\lambda+1 .
\end{aligned}
$$

Subtracting

$$
0=T_{m, m}(\lambda)=\lambda^{2 m+2}-\lambda^{2 m+1}-4 \lambda^{m+1}-\lambda+1,
$$

we obtain

$$
T_{m-k, m+k}(\lambda)=4 \lambda^{m+1}-2 \lambda^{m+k+1}-2 \lambda^{m-k+1}=-2 \lambda^{m-k+1}\left(\lambda^{k}-1\right)^{2}<0 .
$$

Since $\lambda\left(\beta_{m-k, m+k}\right)$ is the largest real root of $T_{m-k, m+k}$, we have $\lambda\left(\beta_{m, m}\right)<$ $\lambda\left(\beta_{m-k, m+k}\right)$.

The other inequalities are proved similarly.

Proposition 3.34 For $m \geq 2$,

$$
\begin{aligned}
\lambda\left(\beta_{m, m}\right) & >\lambda\left(\sigma_{m-1, m+1}\right), \text { and } \\
\lambda\left(\beta_{m, m+1}\right) & \geq \lambda\left(\sigma_{m-1, m+2}\right)
\end{aligned}
$$

with equality if and only if $m=2$.

Proof Let $\lambda=\lambda\left(\sigma_{m-1, m+1}\right)$. Then $T_{m, m}(\lambda)=\lambda^{2 m+2}-\lambda^{2 m+1}-4 \lambda^{m+1}-\lambda+1$. Plugging in the identity

$$
0=S_{m-1, m+1}(\lambda)=\lambda^{2 m+2}-\lambda^{2 m+1}-2 \lambda^{m+2}+2 \lambda^{m}+\lambda-1,
$$

and subtracting this from $T_{m, m}(\lambda)$, we have

$$
T_{m, m}(\lambda)=2 \lambda^{m+2}-4 \lambda^{m+1}-2 \lambda^{m}-2 \lambda+2=2 \lambda^{m}\left(\lambda^{2}-2 \lambda+1\right)+2(1-\lambda) .
$$

The roots of $t^{2}-2 t+1$ are $1 \pm \sqrt{2}$. Since $1-\sqrt{2}<1<\lambda<2<1+\sqrt{2}, \lambda^{2}-2 \lambda+1$ and $1-\lambda$ are both negative, and hence $T_{m, m}(\lambda)<0$. Since $\lambda\left(\beta_{m, m}\right)$ is the largest real root of $T_{m, m}(t)$, it follows that $\lambda\left(\sigma_{m-1, m+1}\right)=\lambda<\lambda\left(\beta_{m, m}\right)$. 
For the second inequality, we plug in $\lambda=\lambda\left(\sigma_{m-1, m+2}\right)$ into $T_{m, m+1}$. This gives

$$
T_{m, m+1}(\lambda)=2 \lambda^{m}\left(\lambda^{3}-\lambda^{2}-\lambda-1\right)-\lambda-1 .
$$

Thus, $\lambda^{3}-\lambda^{2}-\lambda-1<0$ would imply $T_{m, m+1}(\lambda)<0$. The polynomial $g(t)=$ $t^{3}-t^{2}-t-1$ has one real root $(\approx 1.83929)$ and is increasing for $t>1$. Since $\lambda\left(R_{m}\right)$ is decreasing with $m$, and $\lambda<\lambda\left(R_{2}\right) \approx 1.69562<1.8$ by (8), we see that $T_{m, m+1}(\lambda)<0$ for $m \geq 3$. For the remaining case, we check that $T_{2,3}=S_{1,4}$.

Propositions 3.33 and 3.34 show the following.

Corollary 3.35 The least dilatation among $\sigma_{m, n}$ and $\beta_{m, n}$ for $m+n=2 g(g \geq 2)$ is given by $\lambda\left(\sigma_{g-1, g+1}\right)$.

By Corollary 3.23, Lemma 3.29 and Proposition 3.31, the dilatations $\lambda\left(\sigma_{m, n}\right)$ for $n \geq m+2$ converge to 1 as $m, n$ approach infinity. We prove the following stronger statement, which implies Theorems 1.1 and 1.2.

Proposition 3.36 For $g \geq 2$,

$$
\frac{\log (2+\sqrt{3})}{g+1}<\log \left(\lambda\left(\sigma_{g-1, g+1}\right)\right)<\frac{\log (2+\sqrt{3})}{g} .
$$

Proof Using Theorem 3.20, we see that $\lambda=\lambda\left(\sigma_{g-1, g+1}\right)$ satisfies

$$
0=\lambda^{2 g+1}-2 \lambda^{g+1}-2 \lambda^{g}+1=\lambda\left(\lambda^{g}\right)^{2}-2(\lambda+1) \lambda^{g}+1 .
$$

Since $\lambda$ is the largest real solution, the quadratic formula gives

$$
\lambda^{g}=\frac{2(\lambda+1)+\sqrt{4(\lambda+1)^{2}-4 \lambda}}{2 \lambda}=\frac{\lambda+1+\sqrt{\lambda^{2}+\lambda+1}}{\lambda} .
$$

It follows that

$$
\lambda^{g+1}=\lambda+1+\sqrt{\lambda^{2}+\lambda+1} .
$$

Since $1<\lambda<2$ for all $g \geq 2$, (10) implies $2+\sqrt{3}<\lambda^{g+1}<3+\sqrt{7}$.

We improve the upper bound using an argument conveyed to us by Minakawa. Rewrite (9) as follows

$$
0=\lambda^{2 g+1}+\lambda^{2 g}-\lambda^{2 g}-2(\lambda+1) \lambda^{g}+1=\lambda^{2 g}(\lambda+1)-\left(\lambda^{2 g}-1\right)-2(\lambda+1) \lambda^{g} .
$$

Factoring out $(\lambda+1)$ gives

$$
0=\lambda^{2 g}-\frac{\lambda^{2 g}-1}{\lambda+1}-2 \lambda^{g}
$$

Algebraic 83 Geometric Topology, Volume 6 (2006) 
On the other hand, since $\lambda>1$, we have

$$
\frac{\lambda^{2 g}-1}{\lambda+1}<\frac{1}{2}\left(\lambda^{2 g}-1\right) \text {. }
$$

This implies the inequality

$$
x^{2 g}-\frac{x^{2 g}-1}{x+1}-2 x^{g}>x^{2 g}-\frac{1}{2}\left(x^{2 g}-1\right)-2 x^{g}=\frac{1}{2}\left(x^{2 g}-4 x^{g}+1\right)=: p(x)
$$

for $x$ near $\lambda$. Thus, $p(x)$ has a real root $\mu$ larger than $\lambda$. Using the quadratic formula again, we see that $\mu^{g}=2+\sqrt{3}$, and hence $\lambda^{g}<\mu^{g}=2+\sqrt{3}$.

\section{Further discussion and questions}

By Propositions 3.33 and 3.34, for $s \geq 5$ strands, the minimal dilatations by our construction come from $\sigma_{g-1, g+1}$ when $s=2 g+1$; and $\sigma_{g-1, g+2}$ when $s=2 g+2$. For $s$ even, there is an example of a braid with smaller dilatation than that of $\sigma_{g-1, g+2}$ (see the end of Section 4.1), but for $s$ odd, we know of no such examples.

Since $\Sigma(\mathcal{B}(D, 2 g+1)) \subset \Sigma\left(\mathcal{M}_{g}\right)$ (Proposition 2.10), Penner's lower bound [25] for elements of $\Sigma\left(\mathcal{M}_{g}\right)$ extend to $\Sigma(\mathcal{B}(D, 2 g+1))$. Hence we have

$$
\delta(\mathcal{B}(D, 2 g+1)) \geq \delta\left(\mathcal{M}_{g}\right) \geq \frac{\log 2}{12 g-12} .
$$

For $g=2$, Zhirov shows [30] that if $\phi \in \mathcal{M}_{2}$ is pseudo-Anosov with orientable invariant foliations, then $\lambda(\phi)$ is bounded below by the largest root of $x^{4}-x^{3}-x^{2}-x+1$. For $s=5, \sigma_{1,3}$ is pseudo-Anosov, and its lift to $F_{2}$ is orientable. Our formula shows that the dilatation of $\sigma_{1,3}$ is the largest root of Zhirov's equation, and hence $\sigma_{1,3}$ achieves the least dilatation among orientable pseudo-Anosov maps on $F_{2}$. This yields the following weaker version of Ham and Song's result [12], which doesn't assume any conditions on the combinatorics of train tracks.

Corollary 4.1 The braid $\sigma_{1,3}$ is pseudo-Anosov with the least dilatation among braids $\beta \in \mathcal{B}(D ; \mathcal{S})$ on 5 strands such that all singularities of $S^{2} \backslash\left(\mathcal{S} \cup\left\{p_{\infty}\right\}\right)$ for the invariant foliations associated to the pseudo-Anosov map $\Phi_{\widehat{\beta}}$ are even-pronged.

We discuss the following general question and related work on the forcing relation in Section 4.1 .

Question 4.2 Is there a braid $\beta \in \mathcal{B}(D, 2 g+1)$ such that $\lambda(\beta)<\lambda\left(\sigma_{g-1, g+1}\right)$ ? 
Let $\mathcal{K}_{g}^{s} \subset \mathcal{M}_{g}^{s}$ be the subset of mapping classes that arise as the monodromy of a fibered link $(K, F)$ in $S^{3}$, where the fiber $F$ has genus- $g$ and the link $K$ has $s$ components.

Question 4.3 Is there a strict inequality $\delta\left(\mathcal{M}_{g}^{s}\right)<\delta\left(\mathcal{K}_{g}^{s}\right)$ ?

In Section 4.2, we briefly discuss what is known about bounds on dilatations of pseudoAnosov monodromies of fibered links, and show how the braids $\beta_{m, n}$ arise in this class.

\subsection{The forcing relation on the braid types}

The existence of periodic orbits of dynamical systems can imply the existence of other periodic orbits. Continuous maps of the interval give typical examples for such phenomena. Boyland introduced the notion of braid types, and defined a relation on the set of braid types to study an analogous phenomena in the 2-dimensional case. Recall that there is an isomorphism

$$
\mathcal{B}(D ; \mathcal{S}) / Z(\mathcal{B}(D ; \mathcal{S})) \rightarrow M(D ; \mathcal{S}) .
$$

Let $f: D \rightarrow D$ be an orientation preserving homeomorphism with a single periodic orbit $\mathcal{S}$. The isotopy class of $f$ relative to $\mathcal{S}$ is represented by $\beta Z(\mathcal{B}(D ; \mathcal{S}))$ for some braid $\beta \in \mathcal{B}(D ; \mathcal{S})$ by using the isomorphism above. The braid type of $\mathcal{S}$ for $f$, denoted by $b t(\mathcal{S}, f)$, is the conjugacy class $[\beta Z(\mathcal{B}(D ; \mathcal{S}))]$ in the group $\mathcal{B}(D ; \mathcal{S}) / Z(\mathcal{B}(D ; \mathcal{S}))$. To simplify the notation, we will write $[\beta]$ for $[\beta Z(\mathcal{B}(D ; \mathcal{S}))]$. Let

$$
b t(f)=\{b t(P, f) \mid P \text { is a single periodic orbit for } f\},
$$

and $B T$ the set of all braid types for all homeomorphisms of $D$. A relation $\succeq$ on $B T$ is defined as follows: For $b_{i} \in B T(i=1,2)$,

$$
\left.b_{2} \succeq b_{1} \Longleftrightarrow \text { (For any } f: D \rightarrow D \text {, if } b_{2} \in b t(f) \text {, then } b_{1} \in b t(f)\right) \text {. }
$$

We say that $b_{2}$ forces $b_{1}$ if $b_{2} \succeq b_{1}$. It is known that $\succeq$ gives a partial order on $B T$ (see Boyland [6] and Los [21]), and we call the relation the forcing relation.

The topological entropy gives a measure of orbits complexity for a continuous map of the compact space (see Walters [29]). Let $h(f) \geq 0$ be the topological entropy of $f$. For a pseudo-Anosov braid $\beta \in \mathcal{B}(D ; \mathcal{S}), \log (\lambda(\beta))$ is equal to $h(\beta)$, which in turn is the least $h(f)$ among all $f$ with an invariant set $\mathcal{S}$ such that $b t(\mathcal{S}, f)=[\beta]$ (see Fathi-Laudenbach-Poenaru [10, Exposé 10]). One of the relations between the forcing relation and the dilatations is as follows. 
Theorem 4.4 (Los [21]) Let $\beta_{1}$ and $\beta_{2}$ be pseudo-Anosov braids. If $\left[\beta_{2}\right] \succeq\left[\beta_{1}\right]$ and $\left[\beta_{2}\right] \neq\left[\beta_{1}\right]$, then $\lambda\left(\beta_{2}\right)>\lambda\left(\beta_{1}\right)$.

The forcing relation on braids $\beta_{m, n}$ and $\sigma_{m, n}$ was studied by Kin [17].

Theorem 4.5 For any $m, n \geq 1$,

(1) $\left[\beta_{m, n}\right] \succeq\left[\beta_{m, n+1}\right]$,

(2) $\left[\beta_{m, n}\right] \succeq\left[\beta_{m+1, n}\right]$,

(3) $\left[\beta_{m, n}\right] \succeq\left[\sigma_{m, \ell}\right]$ if $\ell \geq m+2$, and

(4) $\left[\sigma_{m, n}\right] \succeq\left[\sigma_{m, \ell}\right]$ if $n \geq \ell \geq m+2$.

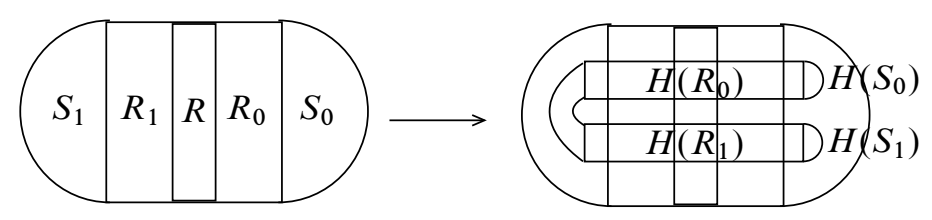

Figure 26: Smale-horseshoe map

The Smale-horseshoe map H: $D \rightarrow D$ is a diffeomorphism such that the action of $\mathrm{H}$ on three rectangles $R_{0}, R_{1}$, and $R$ and two half disks $S_{0}, S_{1}$ is given in Figure 26. The restriction of $\mathrm{H}$ to $R_{i}(i=0,1)$ is an affine map such that $\mathrm{H}$ contracts $R_{i}$ vertically and stretches horizontally. The restriction of $\mathrm{H}$ to $S_{i}(i=0,1)$ is a contraction map. Katok showed [15] that any $C^{1+\epsilon}$ surface diffeomorphism $(\epsilon>0)$ with positive topological entropy has a horseshoe in some iterate. This suggests that the Smale-horseshoe map is a fundamental model for chaotic dynamics.

The set

$$
\Omega=\bigcap_{n \in \mathbb{Z}} \mathrm{H}^{n}\left(R_{0} \cup R_{1}\right)
$$

is invariant under $\mathrm{H}$. Let $\Sigma_{2}=\{0,1\}^{\mathbb{Z}}$, and

$$
\begin{aligned}
\sigma: \Sigma_{2} & \rightarrow \Sigma_{2} \\
\left(\ldots w_{-1} \cdot w_{0} w_{1} \ldots\right) & \mapsto\left(\ldots w_{-1} w_{0} \cdot w_{1} \ldots\right), \quad w_{i} \in\{0,1\}
\end{aligned}
$$

the shift map. There is a conjugacy $\mathcal{K}: \Omega \rightarrow \Sigma_{2}$ between the two maps $\left.H\right|_{\Omega}: \Omega \rightarrow \Omega$ and $\sigma: \Sigma_{2} \rightarrow \Sigma_{2}$ as follows:

$$
\begin{aligned}
\mathcal{K}: \Omega & \rightarrow \Sigma_{2} \\
x & \mapsto\left(\ldots \mathcal{K}_{-1}(x) \mathcal{K}_{0}(x) \mathcal{K}_{1}(x) \ldots\right),
\end{aligned}
$$


where

$$
\mathcal{K}_{i}(x)=\left\{\begin{array}{lll}
0 & \text { if } & \mathrm{H}^{i}(x) \in R_{0} \\
1 & \text { if } & \mathrm{H}^{i}(x) \in R_{1} .
\end{array}\right.
$$

If $x$ is a period $k$ periodic point, then the finite word $\left(\mathcal{K}_{0}(x) \mathcal{K}_{1}(x) \ldots \mathcal{K}_{k-1}(x)\right)$ is called the code for $x$. We say that a braid $\beta$ is a horseshoe braid if there is a periodic orbit for the Smale-horseshoe map whose braid type is $[\beta]$. We define a horseshoe braid type in a similar manner. For the study of the restricted forcing relation on the set of horseshoe braid types, see the papers $[9 ; 11]$ by de Carvalho and Hall.

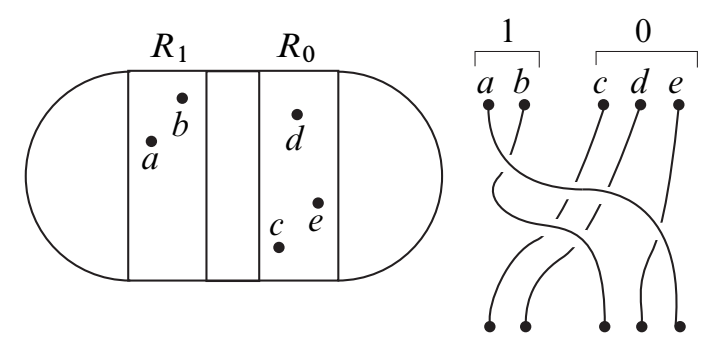

Figure 27: Periodic orbit with the code 10010 and its braid representative

The result by Katok together with Theorem 4.4 implies that horseshoe braids are relevant candidates realizing the least dilatation. It is not hard to see that the braid type of the periodic orbit with the code

$$
1 \underbrace{0 \ldots 0}_{n-1} 1 \underbrace{0 \ldots 0}_{m} \text { or } 1 \underbrace{0 \ldots 0}_{n-1} 1 \underbrace{0 \ldots 0}_{m-1} 1 \quad(n \geq m+2)
$$

is represented by $\left[\sigma_{m, n}^{\prime}\right]\left(=\left[\sigma_{m, n}\right]\right.$ ) (For the definition of $\sigma_{m, n}^{\prime}$, see the end of Section 3.2). Hence, $\sigma_{m, n}(n \geq m+2)$ is a horseshoe braid. Figure 27 illustrates the periodic orbit with the code 10010 and its braid representative.

For the case of even strands, there is a horseshoe braid having dilatation less than our examples. In fact, the braid type of period 8 periodic orbit with the code 10010110 is given by $\left[\beta=\left(\sigma_{1} \sigma_{2} \sigma_{3} \sigma_{4} \sigma_{5} \sigma_{6}\right)^{3} \sigma_{7}\right]$, which satisfies $\lambda(\beta)=1.4134 \ldots<\lambda\left(\sigma_{2,5}\right)=$ $1.5823 \ldots$.

\subsection{Fibered links}

For a fibered link $(K, F)$ with fibering surface $F$, the monodromy $\Phi_{(K, F)}: F \rightarrow F$ is the homeomorphism defined up to isotopy such that the complement of a regular neighborhood of $K$ in $S^{3}$ is a mapping torus for $\Phi_{(K, F)}$. Define $\Delta_{(K, F)}$ to be the characteristic polynomial for the monodromy $\Phi_{(K, F)}$ restricted to first homology 
$\mathrm{H}_{1}(F, \mathbb{R})$. If $K$ is a fibered knot, then $\Delta_{(K, F)}$ is the Alexander polynomial of $K$ (see Kawauchi [16] and Rolfsen [26]).

The homological dilatation of a pseudo-Anosov map $\Phi: F \rightarrow F$ is defined to be $\lambda(f)$, where $f$ is the characteristic polynomial for the restriction of $\Phi$ to $\mathrm{H}_{1}(F ; \mathbb{R})$. Thus, if $(K, F)$ is a fibered link and $\Phi_{(K, F)}$ is the monodromy, then $\lambda\left(\Delta_{(K, F)}\right)$ is the homological dilatation of $\Phi_{(K, F)}$. In the case where $\Phi_{(K, F)}$ is a pseudo-Anosov map, $\lambda\left(\Delta_{(K, F)}\right)$ and $\lambda\left(\Phi_{(K, F)}\right)$ are equal if $\Phi_{(K, F)}$ is orientable (see Rykken [27]).

Any monic reciprocal integer polynomial is equal to $\Delta_{(K, F)}$ for some fibered link $(K, F)$ up to multiples of $(t-1)$ and $\pm t$ (see Kanenobu [14]). In particular, any reciprocal Perron polynomial ${ }^{1}$ can be realized. On the other hand, if $\Phi_{(K, F)}$ is orientable, then $\lambda\left(\Phi_{(K, F)}\right)$ is in general strictly greater than $\lambda\left(\Delta_{(K, F)}\right)$.

Leininger [20] exhibited a pseudo-Anosov map $\Phi_{L}: F_{5} \rightarrow F_{5}$ with dilatation $\lambda_{L}$, where

$$
\log \left(\lambda_{L}\right)=0.162358
$$

A comparison shows that this number is strictly less than our candidate for the least element of $\Sigma(\mathcal{B}(D, 2 g+1)$ for $g=5$ :

$$
\log \left(\lambda\left(\sigma_{4,6}\right)\right)=0.240965 .
$$

The pseudo-Anosov map $\Phi_{L}$ is realized as the monodromy of the fibered $(-2,3,7)-$ pretzel knot. Its dilatation $\lambda_{L}$ is the smallest known Mahler measure greater than 1 among monic integer polynomials (see Boyd [5] and Lehmer [19]).

In the rest of this section, we will construct fibered links whose monodromies are obtained by lifting the spherical mapping classes associated to $\beta_{m, n}$. We set $g=\left\lfloor\frac{m+n}{2}\right\rfloor$. Let $\mathcal{S}$ be the set of marked points on int $(D)$ corresponding to the strands of $\beta_{m, n}$, and $F$ the double covering of $D$, branched over $\mathcal{S}$. Then $F$ has one boundary component if $m+n$ is even and two boundary components if $m+n$ is odd. Let $\Phi_{m, n}^{\prime}$ be the lift of the pseudo-Anosov representative $\Phi_{\beta_{m, n}}$ of $\phi_{\beta_{m, n}} \in \mathcal{M}(D ; \mathcal{S})$ to $F$. Using an argument similar to that in the proof of Proposition 2.10, we have

$$
\lambda\left(\Phi_{m, n}^{\prime}\right)=\lambda\left(\Phi_{\beta_{m, n}}\right)=\lambda\left(\beta_{m, n}\right) .
$$

Note that $\Phi_{m, n}^{\prime}$ is 1 -pronged near each of the boundary of $F$ if $m+n$ is odd.

Let $K_{m, n}$ be the two-bridge link given in Figure 28. By viewing $\left(S^{3}, K_{m, n}\right)$ as the result of a sequence of Hopf plumbings see Hironaka [13, Section 5], one has the following.

\footnotetext{
${ }^{1}$ A monic integer polynomial $f$ is Perron if $f$ has a root $\lambda(f)>1$ such that $\lambda(f)>|\alpha|$ for all roots $\alpha \neq \lambda(f)$.
} 


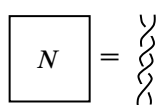

( $N$ positive half twists)

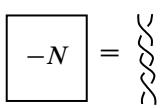

( $N$ negative half twists)

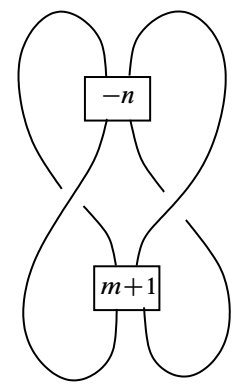

Figure 28: Two-bridge link associated to $\beta_{m, n}$

Proposition 4.6 The complement of a regular neighborhood of $K_{m, n}$ in $S^{3}$ is a mapping torus for $\Phi_{m, n}^{\prime}$.

The fibered links $K_{m, n}$ and the dilatations of $\Phi_{m, n}^{\prime}$ were also studied by Brinkmann [7].

Let $\Delta_{m, n}$ be the Alexander polynomial for $K_{m, n}$. Salem-Boyd sequences for $\Delta_{m, n}$ were computed by Hironaka [13]. Proposition 3.18 implies the following.

Lemma 4.7 If $m$ and $n$ are both odd, then $\lambda\left(\beta_{m, n}\right)=\lambda\left(\Phi_{m, n}^{\prime}\right)=\lambda\left(\Delta_{K_{m, n}}\right)$.

Question 4.8 Let $\Phi_{\sigma_{m, n}}$ be the pseudo-Anosov representative of $\phi_{\sigma_{m, n}}$. Is there a fibered link $K$ in $S^{3}$ such that the complement of a regular neighborhood of $K$ in $S^{3}$ is a mapping torus for a lift of $\Phi_{\sigma_{m, n}}$ ?

\section{References}

[1] M Bauer, An upper bound for the least dilatation, Trans. Amer. Math. Soc. 330 (1992) 361-370 MR1094556

[2] M Bestvina, M Handel, Train-tracks for surface homeomorphisms, Topology 34 (1995) 109-140 MR1308491

[3] JS Birman, Braids, links, and mapping class groups, Princeton University Press, Princeton, N.J. (1974) MR0375281

[4] D W Boyd, Small Salem numbers, Duke Math. J. 44 (1977) 315-328 MR0453692

[5] D W Boyd, Speculations concerning the range of Mahler's measure, Canad. Math. Bull. 24 (1981) 453-469 MR644535 
[6] P Boyland, Rotation sets and monotone periodic orbits for annulus homeomorphisms, Comment. Math. Helv. 67 (1992) 203-213 MR1161281

[7] P Brinkmann, A note on pseudo-Anosov maps with small growth rate, Experiment. Math. 13 (2004) 49-53 MR2065567

[8] A J Casson, S A Bleiler, Automorphisms of surfaces after Nielsen and Thurston, London Mathematical Society Student Texts 9, Cambridge University Press, Cambridge (1988) MR964685

[9] A de Carvalho, T Hall, Braid forcing and star-shaped train tracks, Topology 43 (2004) 247-287 MR2052964

[10] A Fathi, F Laudenbach, V Poenaru, Travaux de Thurston sur les surfaces, Astérisque 66, Société Mathématique de France, Paris (1979) MR568308

[11] T Hall, The creation of horseshoes, Nonlinearity 7 (1994) 861-924 MR1275533

[12] J-Y Ham, W Song, The minimum dilatation of pseudo-Anosov 5-braids, preprint (2005)

[13] E Hironaka, Salem-Boyd sequences and Hopf plumbing, Osaka J. Math. 43 (2006)

[14] T Kanenobu, Module d'Alexander des næuds fibrés et polynôme de Hosokawa des lacements fibrés, Math. Sem. Notes Kobe Univ. 9 (1981) 75-84 MR633997

[15] A Katok, Lyapunov exponents, entropy and periodic orbits for diffeomorphisms, Inst. Hautes Études Sci. Publ. Math. (1980) 137-173 MR573822

[16] A Kawauchi, A survey of knot theory, Birkhäuser Verlag, Basel (1996) MR1417494

[17] E Kin, The forcing partial order on a family of braids forced by pseudo-Anosov 3braids, in preparation

[18] K H Ko, J E Los, W T Song, Entropies of braids, J. Knot Theory Ramifications 11 (2002) 647-666 MR1915500

[19] D H Lehmer, Factorization of certain cyclotomic functions, Ann. of Math. (2) 34 (1933) 461-479 MR1503118

[20] C J Leininger, On groups generated by two positive multi-twists: Teichmüller curves and Lehmer's number, Geom. Topol. 8 (2004) 1301-1359 MR2119298

[21] J Los, On the forcing relation for surface homeomorphisms, Inst. Hautes Études Sci. Publ. Math. (1997) 5-61 MR1471865

[22] T Matsuoka, Braids of periodic points and 2-dimensional analogue of Shorkovskii's ordering, from: "Dynamical systems and Nonlinear Oscillations", (G Ikegami, editor), World Scientific Press (1986) 58-72

[23] C T McMullen, Polynomial invariants for fibered 3-manifolds and Teichmüller geodesics for foliations, Ann. Sci. École Norm. Sup. (4) 33 (2000) 519-560 MR1832823 
[24] H Minakawa, Examples of pseudo-Anosov homeomorphisms with small dilatations, preprint

[25] R C Penner, Bounds on least dilatations, Proc. Amer. Math. Soc. 113 (1991) 443-450 MR1068128

[26] D Rolfsen, Knots and links, Publish or Perish, Berkeley, Calif. (1976) MR0515288

[27] E Rykken, Expanding factors for pseudo-Anosov homeomorphisms, Michigan Math. J. 46 (1999) 281-296 MR1704217

[28] R Salem, A remarkable class of algebraic integers. Proof of a conjecture of Vijayaraghavan, Duke Math. J. 11 (1944) 103-108 MR0010149

[29] P Walters, An introduction to ergodic theory, Graduate Texts in Mathematics 79, Springer, New York (1982) MR648108

[30] A Y Zhirov, On the minimum dilation of pseudo-Anosov diffeomorphisms of a double torus, Uspekhi Mat. Nauk 50 (1995) 197-198 MR1331364

Department of Mathematics, Florida State University

Tallahassee FL 32306-4510, USA

Department of Mathematical and Computing Sciences, Tokyo Institute of Technology

2-12-1-W8-45 Oh-okayama, Meguro-ku, Tokyo 152-8552, Japan

hironaka@math.fsu.edu, kin@is.titech.ac.jp

Received: 23 July 2005 Revised: 13 April 2006 